\title{
Constraints on CEMP-no progenitors from nuclear astrophysics
}

\author{
Arthur Choplin ${ }^{1}$, André Maeder ${ }^{1}$, Georges Meynet ${ }^{1}$, and Cristina Chiappini ${ }^{2}$ \\ ${ }^{1}$ Geneva Observatory, University of Geneva, Maillettes 51, 1290 Sauverny, Switzerland \\ e-mail: arthur .choplin@unige.ch \\ ${ }^{2}$ Leibniz-Institut für Astrophysik Potsdam, An der Sternwarte 16, 14482 Potsdam, Germany \\ Received 6 January 2016 / Accepted 7 June 2016
}

\begin{abstract}
Context. The CEMP-no stars are long-lived small mass stars presenting a very low iron content and overabundances of carbon with no sign or only very weak signs of s- or r-elements. Although the origin of this abundance pattern is still a matter of debate, it was very likely inherited from a previous massive star, which we call the source star.

Aims. We rely on a recent classification of CEMP-no stars arguing that some of them are made of a material processed by hydrogen burning that was enriched in products of helium burning during the nuclear life of the source star. We examine the possibility of forming CEMP-no stars with this material.

Methods. We study the nucleosynthesis of the $\mathrm{CNO}$ cycle and the $\mathrm{Ne}-\mathrm{Na} \mathrm{Mg}$-Al chains in a hydrogen burning single zone while injecting the helium burning products ${ }^{12} \mathrm{C},{ }^{16} \mathrm{O},{ }^{22} \mathrm{Ne}$, and ${ }^{26} \mathrm{Mg}$. We investigate the impact of changing density, temperature and the injection rate. The nuclear reaction rates involving the creation and destruction of ${ }^{27} \mathrm{Al}$ are also examined.

Results. ${ }^{14} \mathrm{~N},{ }^{23} \mathrm{Na},{ }^{24} \mathrm{Mg}$, and ${ }^{27} \mathrm{Al}$ are formed when injecting ${ }^{12} \mathrm{C},{ }^{16} \mathrm{O},{ }^{22} \mathrm{Ne}$, and ${ }^{26} \mathrm{Mg}$ in the hydrogen burning zone. The ${ }^{12} \mathrm{C} /{ }^{13} \mathrm{C}$ ratio is constant under various conditions in the hydrogen burning zone. The predicted $[\mathrm{Al} / \mathrm{Fe}]$ ratio varies up to $\sim 2$ dex depending on the prescription used for the reaction rates involving ${ }^{27} \mathrm{Al}$.

Conclusions. The experiments we carried out support the view that some CEMP-no stars are made of a material processed by hydrogen burning that comes from a massive star experiencing mild to strong rotational mixing. During its burning, this material was likely enriched in helium burning products. No material coming from the carbon-oxygen rich core of the source star should be added to form the daughter star, otherwise the ${ }^{12} \mathrm{C} /{ }^{13} \mathrm{C}$ ratio would be largely above the observed range of values.
\end{abstract}

Key words. nuclear reactions, nucleosynthesis, abundances - stars: chemically peculiar - stars: abundances

\section{Introduction}

The content of iron at the surface of a star is often used as an indication of the chemical enrichment of its environment. A very small amount of iron relative to the sun indicates a region similar to the early universe where only few nucleosynthetic events occurred. A way to obtain new clues on stars in the early universe is then to examine the most iron deficient objects. Carbon-enhanced metal-poor stars (CEMP) are a subclass of iron deficient stars that have an excess of carbon relative to the sun and also an excess of oxygen and nitrogen in general. Although it can vary a bit from one author to another, the two common criteria defining a CEMP are $^{1}[\mathrm{Fe} / \mathrm{H}]<-1.0$ and $[\mathrm{C} / \mathrm{Fe}]>0.7$ (Aoki et al. 2007). The range of $[\mathrm{C} / \mathrm{Fe}]$ covers $\sim 3.5 \mathrm{dex}$, from $[\mathrm{C} / \mathrm{Fe}]=0.7$ to $[\mathrm{C} / \mathrm{Fe}]=4.26$ for $\mathrm{HE} 1327-2326$ (Norris et al. 2013; Allen et al. 2012). The frequency of CEMP seems to rise toward lower [Fe/H] (Lee et al. 2013), but also with increasing distance from the Galactic plane (Frebel et al. 2006) or moving from inner to outer halo (Carollo et al. 2012). The socalled CEMP-no subclass is characterized by its low content in s- or r-elements, contrary to the other sublasses of CEMP stars: CEMP-s, CEMP-r, and CEMP-r/s (Beers \& Christlieb 2005). CEMP-no stars are of particular interest since they dominate at $[\mathrm{Fe} / \mathrm{H}] \lesssim-3$ (Aoki 2010; Norris et al. 2013).

Among the scenarios explored to explain CEMP-no stars, we note the "spinstar" scenario (Meynet et al. 2006, 2010; Hirschi 2007; Chiappini 2013; Maeder et al. 2015) and the

$1[\mathrm{X} / \mathrm{Y}]=\log _{10}\left(N_{\mathrm{X}} / N_{\mathrm{Y}}\right)-\log _{10}\left(N_{\mathrm{X} \odot} / N_{\mathrm{Y} \odot}\right)$ where $N_{\mathrm{X}, \mathrm{Y}}$ is the number density of elements $\mathrm{X}$ and $\mathrm{Y}$, and $\odot$ denotes the abundances in the sun. "mixing and fallback" scenario (Umeda \& Nomoto 2002, 2005; Tominaga et al. 2014). The latter explains CEMP-no with a model of faint supernovae from Pop III stars with mixing and fallback. The mixing considered in these models occurs just before or during the explosion. The zone of mixing and the mass cut $^{2}$ are free parameters of the models adjusted differently in each star to reproduce observed abundance patterns of CEMP-no stars.

According to the spinstar scenario, CEMP-no form in a region previously enriched by material that comes from low metallicity, rotating massive stars. During their nuclear lifetimes, spinstars experience mass loss and strong mixing triggered by rotation. As developed in Maeder et al. (2015), although they are different, these two models appear more complementary than contradictory. Processes like strong internal mixing in the source star, winds, or faint supernova may all have happened. Recently, Takahashi et al. (2014) has presented results based on rotating models with strong fallback (but no mixing in the sense of the works by Umeda \& Nomoto) and tried to deduce the initial rotation of the source stars from comparisons with observed abundance patterns from three CEMP-no stars.

Maeder et al. (2015) proposed the idea that the variety of observed ratios is likely due to material being processed back and forth by hydrogen and helium burning regions in the spinstar. In other words, these two burning regions are exchanging material between them. These exchanges are triggered by the rotational mixing. In a first step, the helium burning products diffuse

\footnotetext{
2 The mass cut delimits the part of the star expelled from the part which is kept in the remnant.
} 

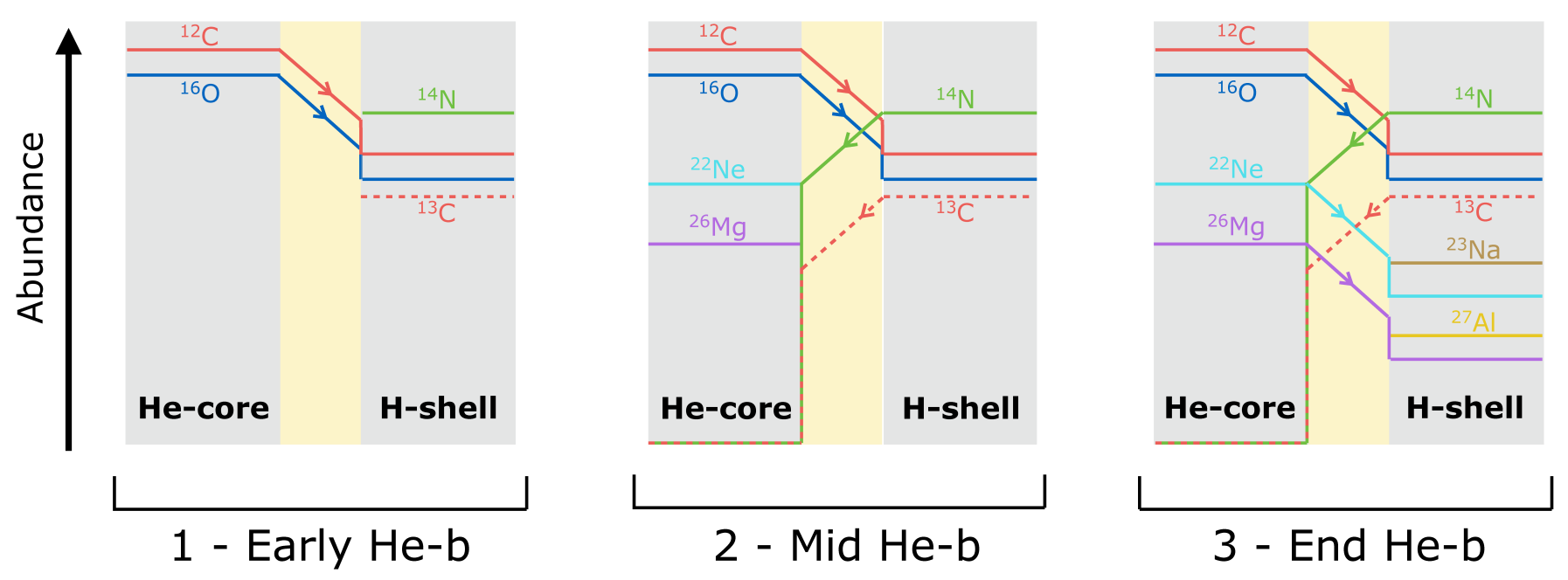

Fig. 1. Schematic view of the "back and forth" process at work in the spinstar. It occurs during the core helium burning phase and it is an exchange of chemical species between the helium burning core and the hydrogen burning shell.

into the hydrogen burning shell. More specifically, ${ }^{12} \mathrm{C}$ and ${ }^{16} \mathrm{O}$ synthesized in the helium core diffuse in the hydrogen burning shell, boosting the $\mathrm{CNO}$ cycle and creating primary ${ }^{14} \mathrm{~N}$ and ${ }^{13} \mathrm{C}$ (see left panel of Fig. 1). In turn, the products of the hydrogen burning shell (among them ${ }^{14} \mathrm{~N}$ ) diffuse back into the helium core. The isotope ${ }^{22} \mathrm{Ne}$ is synthesized through the nuclear chain ${ }^{14} \mathrm{~N}(\alpha, \gamma){ }^{18} \mathrm{~F}\left(, e^{+} v_{e}\right){ }^{18} \mathrm{O}(\alpha, \gamma){ }^{22} \mathrm{Ne}$. The isotope ${ }^{26} \mathrm{Mg}$ can also be synthesized thanks to the reaction ${ }^{22} \mathrm{Ne}(\alpha, \gamma)^{26} \mathrm{Mg}$ (middle panel of Fig. 1). Some ${ }^{25} \mathrm{Mg}$ can also be created through the reaction ${ }^{22} \mathrm{Ne}(\alpha, n)^{25} \mathrm{Mg}$. Neon and magnesium can enter the hydrogen burning shell again, boosting the $\mathrm{Ne}-\mathrm{Na}$ and $\mathrm{Mg}-\mathrm{Al}$ chains and therefore creating sodium and aluminium (right panel of Fig. 1). Through these back and forth exchanges between the hydrogen and helium burning regions, a series of isotopes can be formed. The abundances can vary a lot depending on the strength and number of these exchanges and thus such models can easily account for the variety of the abundance ratios observed at the surface of CEMP-no stars. For a given initial mass and rotation rate, the rotational mixing responsible for the exchanges described above is stronger at low metallicity. This effect is mainly due to the higher compactness of low metallicity stars (Maeder \& Meynet 2001).

Putting aside the complexity of stellar models, we realize in this work simple nuclear experiments in order to illustrate the idea of Maeder et al. (2015) and to constrain the conditions needed in the source stars that would lead to the appropriate nucleosynthesis required to form CEMP-no stars. We study the impact of injecting ${ }^{12} \mathrm{C},{ }^{16} \mathrm{O},{ }^{22} \mathrm{Ne}$, and ${ }^{26} \mathrm{Mg}$ in a hydrogen burning single zone at typical temperatures and densities of the hydrogen burning shell of a $20-60 M_{\odot}$ source star model at very low metallicity $\left(Z=10^{-5}\right)$. Different sets of nuclear rates are tested for the three main reactions involving ${ }^{27} \mathrm{Al}$. We compare our results with a subsample of five CEMP-no stars which have a similar metallicity to that considered in our models and which are, according to Maeder \& Meynet (2015), made of a material processed by hydrogen burning coming from the source star. We note that the active hydrogen burning shell in the source star can be enriched in products of helium burning, as explained previously. Although limited, these numerical experiments, by focusing mainly on the nucleosynthesis of the problem, allow us to explore just what nuclear physics can do and how the results are sensitive to only some nuclear aspects of the problem. As we shall see, even these very simple numerical experiments allow us to obtain very interesting constraints on the sources of CEMP-no stars, constraints that are particularly strong since they are based on the most simple numerical experiments that we can imagine doing.

In Sect. 2, we recall briefly the classification of CEMP-no stars made by Maeder \& Meynet (2015) and select the subsample of CEMP-no stars used in this work. The experiments we carried out are described in Sect. 3 and the results obtained in Sect. 4. Sections 5 and 6 are dedicated to a discussion about the ${ }^{12} \mathrm{C} /{ }^{13} \mathrm{C}$ ratio, the lithium and aluminium abundances. In Sect. 7 , we discuss the possible astronomical origin of the CEMP-no stars considered. Conclusions are given in Sect. 8 .

\section{CEMP-no stars in classes 2 and 4}

Maeder \& Meynet (2015) provided a method for classifying the abundance patterns observed at the surface of CEMP-no stars based on two main ideas: the first is that some material can be exchanged between the hydrogen and helium burning regions inside the star. As noted in the previous section, the hydrogen burning reactions can transform the material enriched in helium burning products. This will boost the abundance of some isotopes, for instance ${ }^{14} \mathrm{~N}$. This ${ }^{14} \mathrm{~N}$ can in turn diffuse into the helium burning region where it is transformed into ${ }^{22} \mathrm{Ne}$, and ${ }^{22} \mathrm{Ne}$ can migrate into the hydrogen burning region, being transformed (at least in part) into ${ }^{23} \mathrm{Na}$. Focusing on hydrogen burning regions, we speak of secular mixing of first order when the $\mathrm{CNO}$ cycle processes material enriched by the normal products of helium burning (typically $\mathrm{C}$ and $\mathrm{O}$ ), and of second order when the nuclear reactions process material enriched in helium burning products resulting from material that was enriched in hydrogen burning products (typically ${ }^{22} \mathrm{Ne}$, resulting from $\alpha$-captures on ${ }^{14} \mathrm{~N}$ ). Similar definitions can be made for the helium burning 
A. Choplin et al.: Constraints on CEMP-no progenitors from nuclear astrophysics

Table 1. Type (MS if $T_{\text {eff }} \geq 5500 \mathrm{~K}$ and $\log g \geq 3.25$, RGB otherwise), class, and abundance data for the CEMP-no stars considered in this work.

\begin{tabular}{crrrrrrrrrrrrr}
\hline Star & Type & Class & {$[\mathrm{Fe} / \mathrm{H}]$} & {$[\mathrm{C} / \mathrm{Fe}]$} & {$[\mathrm{N} / \mathrm{Fe}]$} & {$[\mathrm{O} / \mathrm{Fe}]$} & {$[\mathrm{Na} / \mathrm{Fe}]$} & {$[\mathrm{Mg} / \mathrm{Fe}]$} & {$[\mathrm{Al} / \mathrm{Fe}]$} & {$[\mathrm{Si} / \mathrm{Fe}]$} & {$\left[{ }^{12} \mathrm{C} /{ }^{13} \mathrm{C}\right]$} & $A(\mathrm{Li})$ & $\mathrm{Ref}$ \\
\hline CS 22945-017 & MS & $4++$ & -2.52 & 2.28 & 2.24 & $<2.36$ & - & 0.61 & - & - & -1.17 & $<1.51$ & $4,6,9$ \\
CS 22949-037 & RGB & $4+$ & -3.97 & 1.06 & 2.16 & 1.98 & 2.10 & 1.38 & 0.02 & 0.77 & -1.35 & $<0.13$ & 7,9 \\
CS 22958-042 & MS & 4 & -2.85 & 3.15 & 2.15 & 1.35 & 2.85 & 0.32 & -0.85 & 0.15 & -1.00 & $<1.33$ & $4,6,9$ \\
CS 29498-043 & RGB & 4 & -3.49 & 1.90 & 2.30 & 2.43 & 1.47 & 1.52 & 0.34 & 0.82 & -1.17 & $<-0.05$ \\
CS 30322-023 & RGB & $4++$ & -3.39 & 0.80 & 2.91 & 0.63 & 1.04 & 0.80 & - & 0.58 & -1.35 & $<-0.3$ & $1,4,5,6$ \\
HE 0057-5959 & RGB & $2+\mathrm{Na}$ & -4.08 & 0.86 & 2.15 & $<2.77$ & 1.98 & 0.51 & - & - & $>-1.65$ & - \\
HE 1300+0157 & MS & 2 & -3.75 & 1.31 & $<0.71$ & 1.76 & -0.02 & 0.33 & -0.64 & 0.87 & $>-1.47$ & 1.06 & $2,6,7$ \\
HE 1310-0536 & RGB & $2+$ & -4.15 & 2.36 & 3.20 & $<2.80$ & 0.19 & 0.42 & -0.39 & $<0.25$ & -1.47 & $<0.8$ & 10,11 \\
HE 1327-2326 & MS & $4++$ & -5.76 & 4.26 & 4.56 & 3.70 & 2.48 & 1.55 & 1.23 & - & $>-1.25$ & $<0.62$ & $3,6,7$ \\
HE 1410+0213 & RGB & $2+$ & -2.52 & 2.33 & 2.94 & 2.56 & - & 0.33 & - & - & -1.47 & - & 4,6 \\
HE 1419-1324 & RGB & $4++$ & -3.05 & 1.76 & 1.47 & $<1.19$ & - & 0.53 & - & - & -0.87 & - & 4,6 \\
HE 2331-7155 & RGB & 4 & -3.68 & 1.34 & 2.57 & $<1.70$ & 0.46 & 1.20 & -0.38 & - & -1.25 & - & 11 \\
SMSS 0313-6708 & MS & $4++$ & $<-7.1$ & $>4.50$ & - & - & - & $>3.3$ & - & - & - & 0.7 \\
\hline
\end{tabular}

References. 1 - Masseron et al. (2006); 2 - Frebel et al. (2007); 3 - Frebel et al. (2008); 4 - Masseron et al. (2010); 5 - Masseron et al. (2012); 6 - Allen et al. (2012); 7 - Norris et al. (2013); 8 - Keller et al. (2014); 9 - Roederer et al. (2014); 10 - Hansen et al. (2014); 11 - Hansen et al. (2015).

region. Different families of abundance patterns resulting from hydrogen and helium burning and secular mixing of various orders can result from these back and forth exchanges.

The second idea is that a second type of mixing can be envisaged; this one occurs between the stellar ejecta when the nuclear reactions have stopped. We call this type stellar ejecta mixing. Typically, some CEMP-no stars show signs of being made of material processed by hydrogen and helium burning and then mixed once ejected into the ISM. The material processed by hydrogen burning can result from secular mixing of various orders.

Using these lines of reasoning, Maeder \& Meynet (2015) divided the CEMP-no class in five subclasses.

- Class 0: the CEMP-no is made of a material processed by hydrogen burning, but not enriched in helium burning products (no secular mixing, no mixing of the ejecta).

- Class 1: the CEMP-no is made of a material processed by helium burning, but not enriched in hydrogen burning products (no secular mixing, no mixing of the ejecta).

- Class 2: the CEMP-no is made of a material processed by hydrogen burning enriched in the normal products of helium burning (secular mixing of first order, no mixing of the ejecta).

- Class 3: the CEMP-no is made of a mixture of ejecta involving material processed by both hydrogen and helium burning. The material processed by hydrogen burning results from a secular mixing of first order, and the material processed by helium burning results from a secular mixing of second order (which means that the material processed by helium burning has been enriched by hydrogen burning products that have transformed helium burning products). For instance, large amounts of ${ }^{14} \mathrm{~N}$ and ${ }^{13} \mathrm{C}$ coming from the transformation of ${ }^{12} \mathrm{C}$ and ${ }^{16} \mathrm{O}$, enter the helium core by mixing. Then, successive $\alpha$-captures on ${ }^{14} \mathrm{~N}$ create some ${ }^{22} \mathrm{Ne}$ and ${ }^{25,26} \mathrm{Mg}$.

- Class 4: the CEMP-no is made of a material processed by hydrogen burning (no mixing of the ejecta) resulting from secular mixing of second order. This means that the hydrogen burning transforms material that was processed two times by helium burning. Typically, neon and magnesium enter the hydrogen shell again, boosting the $\mathrm{Ne}-\mathrm{Na}$ and $\mathrm{Mg}-\mathrm{Al}$ chains.

In each of the classes, refinements are made depending on how advanced the nuclear burning is. For instance, the $\mathrm{Mg}$ - $\mathrm{Al}$ chain may have acted to a greater or lesser degree in the source star so that more or less aluminium has been be created. A "+" after the class number indicates a material that is more processed. A material that is even more processed is indicated with " ++ " after the class number. We see that classes 1 and 3 are, at least partly, made of helium burning products while classes 0,2 , and 4 are made of hydrogen burning products. Maeder \& Meynet (2015) attributed a class to 30 out of 46 CEMP-no stars: 4 belonging to class 2, 17 to class 3 , and 9 to class 4 .

In the present work, we focus on CEMP-no stars in classes 0 , 2 , and 4 , i.e. made of a material processed by hydrogen burning that was eventually enriched in helium burning products. A characteristic shared by both stars in classes 2 and 4 (to date there are no observed CEMP-no in class 0) is a relatively low ${ }^{12} \mathrm{C} /{ }^{13} \mathrm{C}$ ratio, between 2 and 12 with a mean of 5.1. This value is characteristic of the CNO processing. The other CEMP-no stars generally have a higher ${ }^{12} \mathrm{C} /{ }^{13} \mathrm{C}$ (up to 50). Part of the helium burning region $\left({ }^{12} \mathrm{C}\right.$-rich and ${ }^{13} \mathrm{C}$-poor) expelled by the source star is used to form classes 1 and 3 , which explains the higher ${ }^{12} \mathrm{C} /{ }^{13} \mathrm{C}$ ratios for class 3 CEMP-no stars (there are no observed CEMP-no in class 1).

Our subsample is finally made of 13 CEMP-no stars in classes 2 and 4 with a mean $[\mathrm{Fe} / \mathrm{H}]$ of -3.9 . Table 1 gives the type, the class, and the abundance data for the sample of CEMP-no stars considered in this work. We note the class $2+\mathrm{Na}$ for HE 0057-5959; "Na" stands because of the high [Na/Fe] ratio. The interpretation is the following: owing to a sufficiently high temperature, a significant amount of ${ }^{20} \mathrm{Ne}$ was synthesized in the helium burning core of the source star. Some of it diffused in the hydrogen burning shell, boosting the $\mathrm{Ne}-\mathrm{Na}$ chain and therefore creating some ${ }^{23} \mathrm{Na}$.

We note that among those 13 CEMP-no stars, 5 are dwarfs (MS, cf. Table 1) and 8 are giants (RGB, cf. Table 1) according to the following criteria: the stars with $T_{\text {eff }} \geq 5500 \mathrm{~K}$ and $\log g \geq 3.25$ are dwarfs, the others are giants. The RGB CEMP may have undergone a dredge-up event, modifying their surface abundances. Such a dredge-up decreases the ${ }^{12} \mathrm{C}$ surface abundances and increases the ${ }^{13} \mathrm{C}$ and ${ }^{14} \mathrm{~N}$ surface abundance. The abundances of $\mathrm{O}, \mathrm{Ne}, \mathrm{Na}, \mathrm{Mg}$, and $\mathrm{Al}$ elements will not change since the temperature inside the hydrogen burning shell of such a low mass star is likely too low to activate the $\mathrm{ON}, \mathrm{Ne}-\mathrm{Na}$, and $\mathrm{Mg}-\mathrm{Al}$ cycles. If we look at the plots $[\mathrm{C} / \mathrm{Fe}]$ and $[\mathrm{N} / \mathrm{Fe}]$ versus $\log g$ for the observed CEMP-no stars (cf. Choplin et al. 2016, Fig. 1) we see that the dispersion of the carbon and nitrogen abundances with respect to iron are quite similar for MS and 
RGB stars. This means that the effect of the first dredge-up does not change the abundance of carbon and nitrogen significantly with respect to the changes related to the dispersion of the initial abundances (about 4 dex). Based on stellar evolution models, Placco et al. (2014) have determined a correction

$\Delta[\mathrm{C} / \mathrm{Fe}]=[\mathrm{C} / \mathrm{Fe}]_{\text {ini }}-[\mathrm{C} / \mathrm{Fe}]_{\text {after } 1 \mathrm{DUp}}$

to apply to the $[\mathrm{C} / \mathrm{Fe}]$ ratio of 505 metal-poor stars in order to recover their initial $[\mathrm{C} / \mathrm{Fe}]$. This correction corresponds to the effect of the first dredge-up (if any). Any dredge-up would decrease $[\mathrm{C} / \mathrm{Fe}]$ so that $\Delta[\mathrm{C} / \mathrm{Fe}] \geq 0$. We note that $\Delta[\mathrm{C} / \mathrm{Fe}]=0$ for MS CEMP since they likely did not experience the first dredge-up. Five out of the eight RGB CEMP considered here belong to this sample; three have $\Delta[\mathrm{C} / \mathrm{Fe}]<0.1$, one $\Delta[\mathrm{C} / \mathrm{Fe}]=$ 0.31 (CS 29498-043), and one $\Delta[\mathrm{C} / \mathrm{Fe}]=0.74$ (CS 22949-037). These corrections are small compared to the observed range of $[\mathrm{C} / \mathrm{Fe}]$ ratios.

In addition, because the $\mathrm{CNO}$ equilibrium value of ${ }^{12} \mathrm{C} /{ }^{13} \mathrm{C}$ is obtained at the surface of the MS stars, this implies that such low values, at least in these stars, cannot be due to a dredge-up event. Moreover, the highest ${ }^{12} \mathrm{C} /{ }^{13} \mathrm{C}$ belong to HE $1419-1324$, a RGB CEMP, showing that the RGB feature is not necessarily associated with a low ${ }^{12} \mathrm{C} /{ }^{13} \mathrm{C}$ ratio, as expected by the effect of the dredge-up. Correcting the $\mathrm{CNO}$ abundances of the evolved CEMP-no stars is of course important in general, but in the framework of the present work we focus on the range of observed abundances rather than of individual stars, and hence these small corrections have no impact on our conclusions.

\section{Presentation of the experiment}

The conducted experiment consists in injecting products synthesized in the helium burning core of massive stars like ${ }^{12} \mathrm{C},{ }^{16} \mathrm{O}$, ${ }^{22} \mathrm{Ne}$, or ${ }^{26} \mathrm{Mg}$ in a hydrogen burning single zone of $1 M_{\odot}$ (hereafter H-box) with a constant temperature $T$ and density $\rho$. The $H$-box schematically reproduces the convective hydrogen burning shell during the core helium burning phase of the source star.

To set the initial conditions in the H-box, we rely on a rotating $60 M_{\odot}$ model computed with the Geneva code. The initial metallicity is $Z=10^{-5}$. It corresponds to $[\mathrm{Fe} / \mathrm{H}]=-3.8$ for the initial mixture we considered ( $\alpha$-enhanced). The initial abundances in the H-box are taken from the hydrogen burning shell of this model, at the beginning of the core helium burning phase, when the mass fraction of ${ }^{4} \mathrm{He}$ in the core $X_{\mathrm{c}}\left({ }^{4} \mathrm{He}\right)=0.98$. Figure 2 shows the temperature and density profiles of this model during the core helium burning phase. The variable $M_{r}$ is the mass coordinate and $X_{\mathrm{c}}\left({ }^{4} \mathrm{He}\right)$ the central mass fraction of ${ }^{4} \mathrm{He}$. We see from this figure that $T$ and $\rho$ in the convective hydrogen burning shell take values of $30-80 \mathrm{MK}$ and $1-5 \mathrm{~g} \mathrm{~cm}^{-3}$, respectively. For a $20 M_{\odot}$ model, the ranges of $T$ and $\rho$ are 30-60 MK and $1-10 \mathrm{~g} \mathrm{~cm}^{-3}$. As a first step, we fix $T=50 \mathrm{MK}$ and $\rho=1 \mathrm{~g} \mathrm{~cm}^{-3}$ in the H-box. Different temperatures and densities in the H-box are discussed later. The simulation is stopped either when the hydrogen in the box is exhausted (when the mass fraction of hydrogen in the H-box $\left.X\left({ }^{1} \mathrm{H}\right)<10^{-8}\right)$ or when the time $t$ exceeds 10 Myr. We note that depending on the stellar model chosen for setting the initial abundances in the H-box, we can have a slightly different initial chemical composition in the H-box. This will depend on the chemical composition of the model in its hydrogen burning shell, at the core helium burning ignition $\left(X_{\mathrm{c}}\left({ }^{4} \mathrm{He}\right)=0.98\right)$. The CNO abundances in the hydrogen burning shell at the core helium burning ignition do not change significantly from a $20 M_{\odot}$ to a $60 M_{\odot}$ model. The abundances of neon, sodium, magnesium, and aluminium do vary a
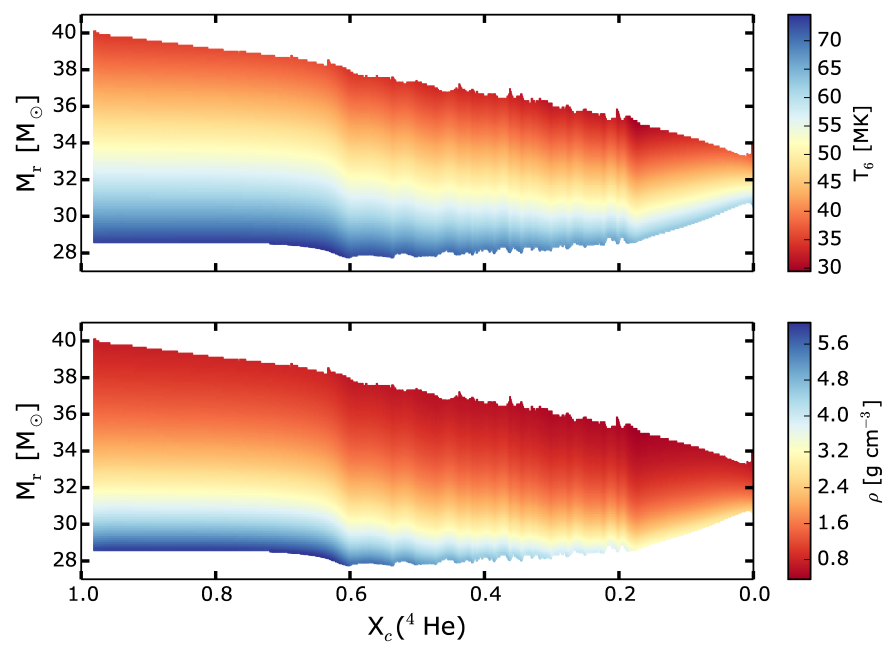

Fig. 2. $M_{r}$ as a function of $X_{\mathrm{c}}\left({ }^{4} \mathrm{He}\right)$, the central mass fraction of ${ }^{4} \mathrm{He}$ for a rotating $60 M_{\odot}$ model at a metallicity $Z=10^{-5}$ (similar to a Kippenhahn diagram). Shown in color is the temperature $T_{6}$ in MK (upper panel) and the density $\rho$ in $\mathrm{g} \mathrm{cm}^{-3}$ (lower panel) in the convective hydrogen burning shell during the core helium burning phase from $X_{\mathrm{c}}\left({ }^{4} \mathrm{He}\right)=$ 0.98 to $X_{\mathrm{c}}\left({ }^{4} \mathrm{He}\right)=0$.

bit more owing to the difference in temperature that implies a slightly different nucleosynthesis in the hydrogen burning shell at the very beginning of the core helium burning phase.

Regarding the nuclear reaction rates, we took the ones used in the Geneva code (see Ekström et al. 2012). These rates are mainly from Angulo et al. (1999) for the CNO cycle, but almost all rates for $\mathrm{Ne}-\mathrm{Na} \mathrm{Mg}-\mathrm{Al}$ chains are from Hale et al. (2002). Only ${ }^{20} \mathrm{Ne}(p, \gamma){ }^{21} \mathrm{Na}$ and ${ }^{21} \mathrm{Ne}(p, \gamma){ }^{22} \mathrm{Na}$ are taken from Angulo et al. (1999) and Iliadis et al. (2001), respectively. We note that the final abundance of ${ }^{26} \mathrm{Al}$ in the $\mathrm{H}$-box is added to the one of ${ }^{26} \mathrm{Mg}$ since ${ }^{26} \mathrm{Al}$ is a radioactive isotope $\left(t_{1 / 2}=\right.$ $7.17 \times 10^{5} \mathrm{yrs}$ ) decaying into ${ }^{26} \mathrm{Mg}$.

In order to reproduce schematically the diffusion of ${ }^{12} \mathrm{C}$, ${ }^{16} \mathrm{O},{ }^{22} \mathrm{Ne}$, and ${ }^{26} \mathrm{Mg}$ from the helium core to the hydrogen shell, we inject a constant mass per year into the H-box, which comes from a reservoir composed only of the considered species $\left({ }^{12} \mathrm{C},{ }^{16} \mathrm{O},{ }^{22} \mathrm{Ne}\right.$, or $\left.{ }^{26} \mathrm{Mg}\right)$. We consider injection rates of $10^{-10}$, $10^{-8}$, and $10^{-6} M_{\odot} \mathrm{yr}^{-1}$ for ${ }^{12} \mathrm{C}$ and ${ }^{16} \mathrm{O}$ and $10^{-12}, 10^{-10}$, and $10^{-8} M_{\odot} \mathrm{yr}^{-1}$ for ${ }^{22} \mathrm{Ne}$ and ${ }^{26} \mathrm{Mg}$. More details about the method for injecting the species and the justification of the adopted injection rates are given in the Appendix. Four cases are tested in the present experiment:

- no injection is made in the H-box;

- ${ }^{12} \mathrm{C}$ and ${ }^{16} \mathrm{O}$ are injected;

$-{ }^{12} \mathrm{C},{ }^{16} \mathrm{O}$, and ${ }^{22} \mathrm{Ne}$ are injected;

$-{ }^{12} \mathrm{C},{ }^{16} \mathrm{O},{ }^{22} \mathrm{Ne}$, and ${ }^{26} \mathrm{Mg}$ are injected.

We note that in a real star, the mass is conserved and thus any injection into the hydrogen burning shell implies that some matter has to diffuse away from that region. In complete stellar models, the elements that are more abundant in the hydrogen burning shell than in one of the two adjacent regions will diffuse into the region(s) where this element is less abundant. However to keep the model as simple as possible we do not consider that complication here. The present work can be seen as a numerical experiment and not as an attempt to model all the details of what happens in stars. Indeed, the most important gradients of abundances are those from the difference in the abundances between the helium core and the hydrogen burning shell. Diffusion from 


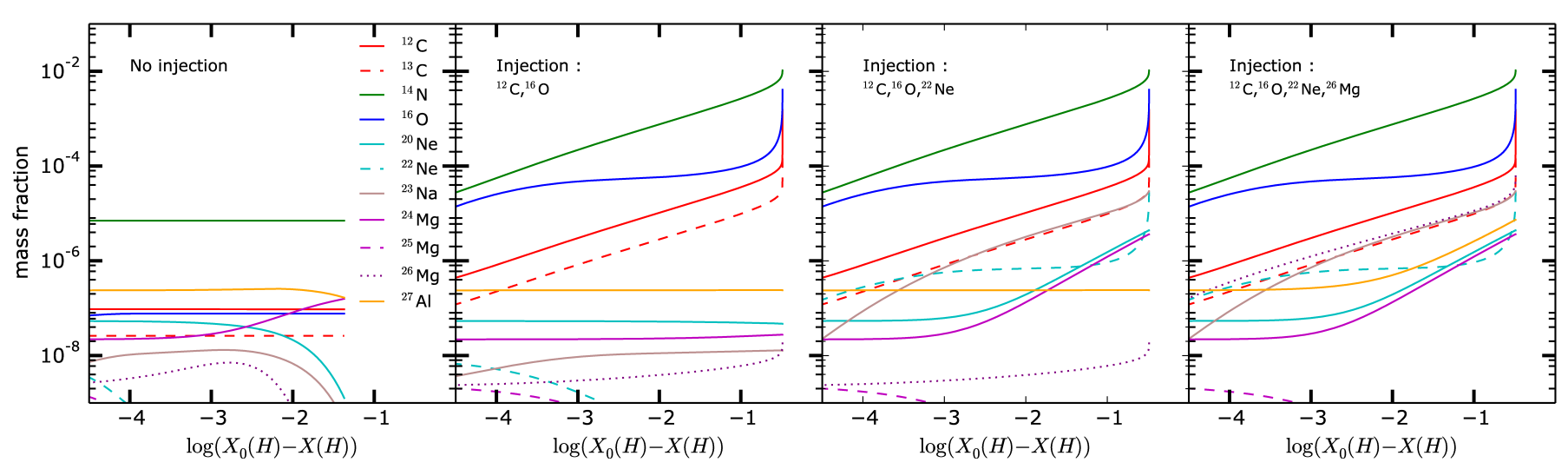

Fig. 3. Abundances in the H-box as a function of the logarithm of $X_{0}(\mathrm{H})-X(\mathrm{H})$. The four panels (from left to right) correspond to the four considered cases: when no injection is made; when ${ }^{12} \mathrm{C}$ and ${ }^{16} \mathrm{O}$ are injected; when ${ }^{12} \mathrm{C},{ }^{16} \mathrm{O}$, and ${ }^{22} \mathrm{Ne}$ are injected; and when ${ }^{12} \mathrm{C},{ }^{16} \mathrm{O},{ }^{22} \mathrm{Ne}$, and ${ }^{26} \mathrm{Mg}$ are injected. Density and temperature in the box are $\rho=1 \mathrm{~g} \mathrm{~cm}^{-3}$ and $T_{6}=50 \mathrm{MK}$

the helium core to the hydrogen burning shell is therefore clearly the dominant feature, blurring all the other diffusion processes In complete stellar models, injection of nitrogen into the helium core occurs dominantly by convection when the helium core slightly grows in regions left over by the hydrogen burning shell, not by diffusion from the hydrogen to the helium burning region. Also, as we show, the results obtained from complete stellar models (that follow in a consistent way the mixing of the elements) are well reproduced by our simple box model. Thus we are quite confident that our simple approach grasps the essentials of the process.

We also note that the origin of the iron is not investigated in the present work. By choosing a non-zero metallicity, we assume that the small iron content observed at the surface of the CEMP-no stars is already present in the source star (and in the H-box). Our sample of class 2 and 4 CEMP-no stars is expected to be made of a material processed by hydrogen burning that comes from the source star, i.e. from the outer layers of this star. The iron abundance in those outer layers is likely not affected by the nucleosynthesis and remains equal to its initial value. As a consequence, a comparison of the models with the observed ratios like $[\mathrm{C} / \mathrm{Fe}]$ or $[\mathrm{N} / \mathrm{Fe}]$ can be made, provided that the iron content in the models (the $[\mathrm{Fe} / \mathrm{H}]$ ratio) is similar to the iron content of the CEMP-no stars. In our models, $[\mathrm{Fe} / \mathrm{H}]=-3.8$ so that the observed CEMP-no stars around this value can be consistently compared with the models.

\section{Results of the experiment}

Figure 3 shows the mass fraction of elements as a function of $\log \left(X_{0}(\mathrm{H})-X(\mathrm{H})\right)$ (i.e. the logarithm of the initial mass fraction of hydrogen minus the current hydrogen mass fraction in the H-box) for the four cases presented in Sect. 3. The initial mass fraction of ${ }^{1} \mathrm{H}$ in the box is equal to 0.33 , the temperature and density are set to $T=50 \mathrm{MK}$ and $\rho=1 \mathrm{~g} \mathrm{~cm}^{-3}$, and the injection rates are $10^{-8} M_{\odot} \mathrm{yr}^{-1}$ for ${ }^{12} \mathrm{C}$ and ${ }^{16} \mathrm{O}$ and $10^{-10} M_{\odot} \mathrm{yr}^{-1}$ for ${ }^{22} \mathrm{Ne}$ and ${ }^{26} \mathrm{Mg}$. We describe now the four panels in Fig. 3, from left to right:

1. When no injection is made only $4.28 \times 10^{-2}$ of hydrogen (in mass fraction) is consumed at the end of the limited time, which we fixed at $10 \mathrm{Myr}$. No transient regime is seen for the $\mathrm{CNO}$ elements since the $\mathrm{CNO}$ cycle is already at equilibrium at $t=0$. The initial increase of $X\left({ }^{23} \mathrm{Na}\right)$, the mass fraction of sodium, is due to the effect of the
$\mathrm{Ne}-\mathrm{Na}$ chain. It finally drops, like $X\left({ }^{20} \mathrm{Ne}\right)$, in favour of $X\left({ }^{24} \mathrm{Mg}\right)$ owing to the reaction ${ }^{23} \mathrm{Na}(p, \gamma){ }^{24} \mathrm{Mg}$. At $T_{6}=$ 50 , since the ${ }^{24} \operatorname{Mg}(p, \gamma){ }^{25} \mathrm{Al}$ reaction is weak, it does not transform efficiently the ${ }^{24} \mathrm{Mg}$ synthesized so that the ${ }^{24} \mathrm{Mg}$ abundance increases. A small amount of ${ }^{27} \mathrm{Al}$ is destroyed when little ${ }^{1} \mathrm{H}$ remains in the shell and is transformed either into ${ }^{28} \mathrm{Si}$ through ${ }^{27} \mathrm{Al}(p, \gamma){ }^{28} \mathrm{Si}$, or into ${ }^{24} \mathrm{Mg}$ through ${ }^{27} \mathrm{Al}(p, \alpha){ }^{24} \mathrm{Mg}$, both channels being almost equal at this temperature.

2. When ${ }^{12} \mathrm{C}$ and ${ }^{16} \mathrm{O}$ are injected, the $\mathrm{CNO}$ cycle is boosted and primary ${ }^{14} \mathrm{~N}$ and ${ }^{13} \mathrm{C}$ are created. The $\mathrm{CNO}$ elements are more and more abundant so that more and more hydrogen is burnt. It is finally exhausted after $\sim 0.8 \mathrm{Myr}$. The reactions in the $\mathrm{CN}$ cycle are fast so that an equilibrium is almost instantaneously reached for ${ }^{12} \mathrm{C},{ }^{13} \mathrm{C}$, and ${ }^{14} \mathrm{~N}$. Since the reaction ${ }^{16} \mathrm{O}(p, \gamma){ }^{17} \mathrm{~F}$ is much slower, the injected ${ }^{16} \mathrm{O}$ accumulates before being transformed into ${ }^{17} \mathrm{~F}$. These two regimes can be seen in the second panel of Fig. 3: the curve showing ${ }^{16} \mathrm{O}$ first increases until $\log \left(X_{0}(\mathrm{H})-X(\mathrm{H})\right) \sim-3$ (accumulation) and then becomes flatter for $-3<\log \left(X_{0}(\mathrm{H})-X(\mathrm{H})\right)<-1$ $\left({ }^{16} \mathrm{O}\right.$ destruction becomes important). No permanent regime is attained for ${ }^{16} \mathrm{O}$. At the end ${ }^{12} \mathrm{C}$ and ${ }^{16} \mathrm{O}$ rise dramatically because the hydrogen is almost exhausted: the CNO cycle works less and less, implying an accumulation of the injected ${ }^{12} \mathrm{C}$ and ${ }^{16} \mathrm{O}$. Regarding the other elements, we see that ${ }^{22} \mathrm{Ne}$ decreases in favour of ${ }^{23} \mathrm{Na}$, but the duration of the simulation is too short in this case for the $\mathrm{Ne}-\mathrm{Na}$ and $\mathrm{Mg}-\mathrm{Al}$ chains to operate significantly.

3. When the same experiment is performed and some ${ }^{22} \mathrm{Ne}$ is also injected, ${ }^{23} \mathrm{Na}$ and ${ }^{24} \mathrm{Mg}$ are synthesized through the reactions ${ }^{22} \mathrm{Ne}(p, \gamma)^{23} \mathrm{Na}$ and ${ }^{23} \mathrm{Na}(p, \gamma)^{24} \mathrm{Mg}$. Some ${ }^{20} \mathrm{Ne}$ is also created when the reaction ${ }^{23} \mathrm{Na}(p, \alpha){ }^{20} \mathrm{Ne}$ occurs. At this temperature and for the selected rates, the $(p, \alpha)$ channel is 1.6 higher than the $(p, \gamma)$ channel so that ${ }^{23} \mathrm{Na}$ is almost equally destroyed in ${ }^{20} \mathrm{Ne}$ and ${ }^{24} \mathrm{Mg}$. The reaction ${ }^{24} \mathrm{Mg}(p, \alpha){ }^{25} \mathrm{Al}$ is too slow to activate the $\mathrm{Mg}$-Al chain. Since neon, sodium, and magnesium are much less abundant than the CNO elements, the hydrogen is not burnt significantly quicker than in case 2 when only ${ }^{12} \mathrm{C}$ and ${ }^{16} \mathrm{O}$ are injected.

4. When injecting ${ }^{26} \mathrm{Mg}$ as well there is a $\sim 2$ dex increase in ${ }^{27} \mathrm{Al}$ due to the direct transformation of ${ }^{26} \mathrm{Mg}$ into ${ }^{27} \mathrm{Al}$ thanks to a proton capture. For the temperature, density, and time of simulation considered, the ${ }^{27} \mathrm{Al}$ destruction through either the $(p, \alpha)$ or $(p, \gamma)$ channels is not significant. 


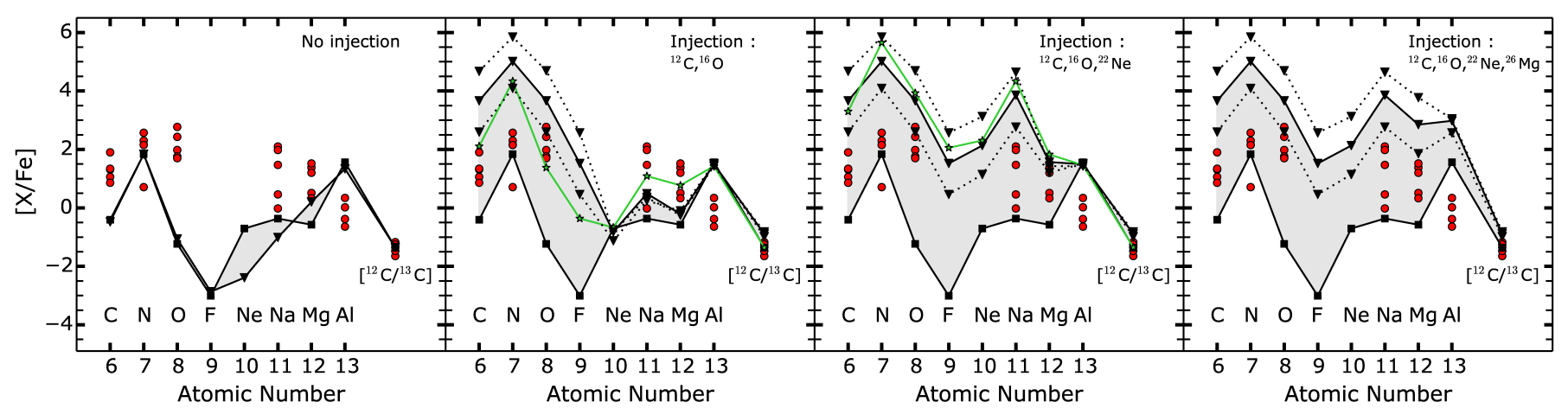

Fig. 4. $[\mathrm{X} / \mathrm{Fe}]$ ratios in the H-box for the four considered cases (from left to right). The $\left[{ }^{12} \mathrm{C} /{ }^{13} \mathrm{C}\right]$ ratio is also shown (i.e. ${ }^{12} \mathrm{C} /{ }^{13} \mathrm{C}$ ratio relative to the sun, in logarithm). The red points show the CEMP-no stars of Table 1 with $[\mathrm{Fe} / \mathrm{H}]=-3.8 \pm 0.3$. The abundance patterns in the box at $t=0$ are represented by black lines with squares and the patterns at the end of the simulation by black lines with triangles. The lower and upper black dotted lines represent the final composition in the H-box when the rates of injection are divided and multiplied by $10^{2}$, respectively. The green pattern on the second (third) panel shows the composition in the hydrogen burning shell at the end of the core helium burning phase of a complete $20 M_{\odot}$ stellar model at $30 \%(70 \%)$ of the critical velocity on the ZAMS. Density and temperature in the box are $\rho=1 \mathrm{~g} \mathrm{~cm}^{-3}$ and $T_{6}=50 \mathrm{MK}$.

Figure 4 shows the $[\mathrm{X} / \mathrm{Fe}]$ ratios for the four cases presented in Fig. 3. The initial abundance pattern in the H-box (lines with squares) and the final pattern (lines with triangles) are plotted. The grey area corresponds to the range of values covered during the simulation. The dotted lines correspond to the final patterns in the H-box when the rates of injection are divided and multiplied by 100 . The red points represent the observed ratios in CEMP-no stars of similar metallicities $([\mathrm{Fe} / \mathrm{H}]=-3.8 \pm 0.3)$ to the metallicity considered in our numerical experiment $([\mathrm{Fe} / \mathrm{H}]=$ -3.8). As mentioned in Sect. 3, it is important to focus on the CEMP-no stars that have similar metallicities to the one in the models since the iron abundance in our model is an initial condition that is not modified by the nuclear reactions occurring in the $\mathrm{H}$-box. Any change in the initial iron abundance in the models will produce a shift of the $[\mathrm{X} / \mathrm{Fe}]$ ratios predicted by the models.

Without injection, the abundance pattern in the H-box does not change very much and all the $[\mathrm{X} / \mathrm{Fe}]$ ratios (except $[\mathrm{N} / \mathrm{Fe}]$ and $[\mathrm{Al} / \mathrm{Fe}])$ stay below the observed values. This is not surprising since the observed values correspond to classes 2 and 4 , while the present experiment (no injection) would instead correspond to the CEMP-no stars in class 0 . This class is made of a material processed only by hydrogen burning and where no mixing occurred between the hydrogen and helium burning regions. Injecting some ${ }^{12} \mathrm{C}$ and ${ }^{16} \mathrm{O}$ enhances the corresponding $[\mathrm{X} / \mathrm{Fe}]$ ratios and the $[\mathrm{N} / \mathrm{Fe}]$ (Fig. 4, second panel). Increasing or decreasing the rate of injection by a factor of 100 (dotted lines) changes the final pattern in the box, but not dramatically: from the lower to the upper dotted line, the rate of injection is multiplied by 4 dex while the difference in $[\mathrm{X} / \mathrm{Fe}]$ values does not exceed 2 dex (for $\mathrm{C}, \mathrm{N}$, and $\mathrm{O}$ ) because when injecting more ${ }^{12} \mathrm{C}$ and ${ }^{16} \mathrm{O}$ in the $\mathrm{H}$-box, the hydrogen is burnt more rapidly and hydrogen exhaustion occurs earlier. We have here a negative feedback process: increasing the rate of injection increases the amount of injected species, but at the same time reduces the available time for injecting those new chemical species in the $\mathrm{H}$-box. This explains qualitatively why injecting ${ }^{12} \mathrm{C}$ and ${ }^{16} \mathrm{O}$ at a rate $10^{4}$ higher does not lead to an increase of 4 dex in the final $[\mathrm{C} / \mathrm{Fe}],[\mathrm{N} / \mathrm{Fe}]$, and $[\mathrm{O} / \mathrm{Fe}]$ ratios. The third case shows enhancements of $[\mathrm{Ne} / \mathrm{Fe}],[\mathrm{Na} / \mathrm{Fe}]$, and $[\mathrm{Mg} / \mathrm{Fe}]$ ratios: protons captured on the injected ${ }^{22} \mathrm{Ne}$ create ${ }^{23} \mathrm{Na}$ and then ${ }^{24} \mathrm{Mg}$. The final patterns of the fourth case present enhancements of $[\mathrm{Mg} / \mathrm{Fe}]$ and $[\mathrm{Al} / \mathrm{Fe}]$ ratios compared to case 3: owing to the injection of ${ }^{26} \mathrm{Mg}$, the $\mathrm{Mg}-\mathrm{Al}$ chain is boosted, hence creating some ${ }^{27} \mathrm{Al}$.
Changing the temperature and the density in the H-box leads to the results presented in Fig. 5. We tested temperatures of $T_{6}=$ 50, 60, and $80 \mathrm{MK}$ at a constant density $\rho=1 \mathrm{~g} \mathrm{~cm}^{-3}$ (left panel) and densities of $\rho=1,10$, and $100 \mathrm{~g} \mathrm{~cm}^{-3}$ at a constant temperature $T_{6}=50 \mathrm{MK}$ (right panel). For both cases, the injected species are ${ }^{12} \mathrm{C},{ }^{16} \mathrm{O},{ }^{22} \mathrm{Ne}$, and ${ }^{26} \mathrm{Mg}$ and the injection rates are $10^{-8} M_{\odot} \mathrm{yr}^{-1}$ for ${ }^{12} \mathrm{C}$ and ${ }^{16} \mathrm{O}$ and $10^{-10} M_{\odot} \mathrm{yr}^{-1}$ for ${ }^{22} \mathrm{Ne}$ and ${ }^{26} \mathrm{Mg}$. In addition to the other [X/Fe] ratios, $[\mathrm{Si} / \mathrm{Fe}]$ is also shown. Increasing either the temperature or the density leads to lower $[\mathrm{X} / \mathrm{Fe}]$ ratios at hydrogen exhaustion (except for the $[\mathrm{Si} / \mathrm{Fe}]$ ratio at $\left.T_{6}=80 \mathrm{MK}\right)$. When raising the density for instance, the rates of the nuclear reactions increase allowing a quicker synthesis of the chemical species than at lower densities. At the same time, the hydrogen is burnt more rapidly so that hydrogen exhaustion occurs earlier, leaving less time to inject new species. Summing those two opposite effects finally leads to lower $[\mathrm{X} / \mathrm{Fe}]$ ratios at hydrogen exhaustion. For the same reasons, similar results are found when varying the temperature, although the dependance of the nuclear rates on temperature is much stronger than the dependance on the density. This is the reason why the various temperatures spanning a relatively small range of values (see Fig. 5) change the $[\mathrm{X} / \mathrm{Fe}]$ ratios much more significantly than the various densities, even though they cover a much larger range of values.

The initial $[\mathrm{Si} / \mathrm{Fe}]$ ratio in the box is about 1 and it is little affected by changes in temperature and density. However, increasing the temperature to $T_{6}=80 \mathrm{MK}$ leads to about $0.5 \mathrm{dex}$ more silicon at the end. The first reason is that the nuclear rates associated with $\mathrm{Mg}, \mathrm{Al}$, and $\mathrm{Si}$ are generally 3-4 dex higher at $T_{6}=80 \mathrm{MK}$ than at $T_{6}=50 \mathrm{MK}$, allowing the synthesis of some ${ }^{28} \mathrm{Si}$. The second reason is that the ${ }^{27} \mathrm{Al}$ is destroyed either to form ${ }^{28} \mathrm{Si}$ thanks to the ${ }^{27} \mathrm{Al}(p, \gamma)^{28} \mathrm{Si}$ reaction or to form ${ }^{24} \mathrm{Mg}$ owing to the reaction ${ }^{27} \mathrm{Al}(p, \alpha){ }^{24} \mathrm{Mg}$. When ${ }^{27} \mathrm{Al}$ transforms into ${ }^{24} \mathrm{Mg}$, the $\mathrm{Mg}$-Al chain operates and synthesizes ${ }^{27} \mathrm{Al}$ again. Instead, ${ }^{27} \mathrm{Al}$ is definitely destroyed when it is transformed into ${ }^{28} \mathrm{Si}$. At $T_{6}=50$ and $60 \mathrm{MK}$, both channels are roughly equal. At $T_{6}=80 \mathrm{MK}$, the $(p, \gamma)$ channel is $\sim 40$ times higher than the $(p, \alpha)$ channel. This tends to reduce $\mathrm{Mg}$ and $\mathrm{Al}$ and to increase $\mathrm{Si}$.

Also shown in Figs. 4 and 5 is the $\left[{ }^{12} \mathrm{C} /{ }^{13} \mathrm{C}\right]$ ratio, i.e. $\log \left({ }^{12} \mathrm{C} /{ }^{13} \mathrm{C}\right)-\log \left({ }^{12} \mathrm{C} /{ }^{13} \mathrm{C}\right)_{\odot}$. The isotopic ratio in the Sun is taken from Lodders (2003). In all cases, the $\left[{ }^{12} \mathrm{C} /{ }^{13} \mathrm{C}\right]$ ratio does not vary more than 0.5 dex, always staying around -1.5 because 


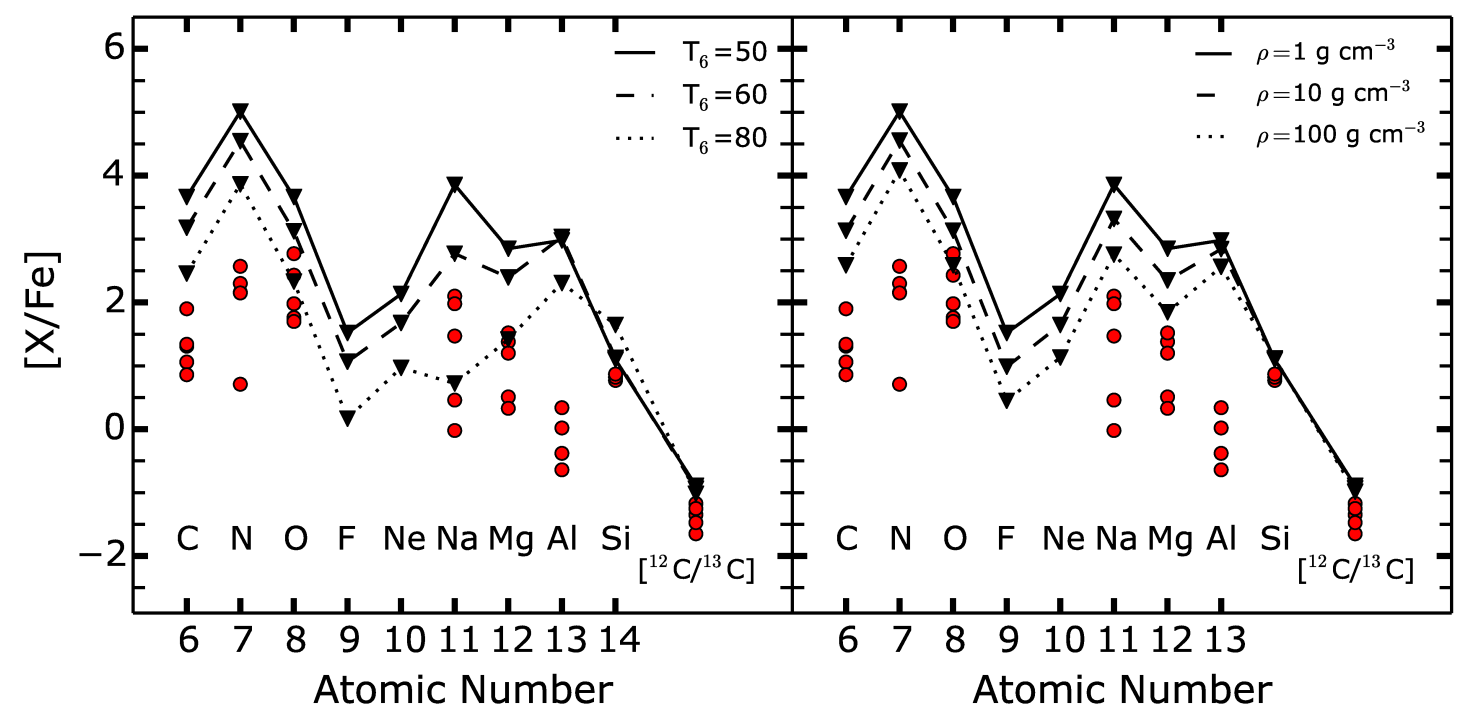

Fig. 5. Left: same as Fig. 4 but for three different temperatures in the H-box. The [Si/Fe] ratio is also shown. Only the final abundance patterns in the box are plotted. The injected species are ${ }^{12} \mathrm{C},{ }^{16} \mathrm{O},{ }^{22} \mathrm{Ne}$, and ${ }^{26} \mathrm{Mg}$. Density is unchanged $\left(1 \mathrm{~g} \mathrm{~cm}^{-3}\right)$ and the injection rate is $10^{-8} M_{\odot} \mathrm{yr}^{-1}$ for ${ }^{12} \mathrm{C}$ and ${ }^{16} \mathrm{O}$ and $10^{-10} M_{\odot} \mathrm{yr}^{-1}$ for ${ }^{22} \mathrm{Ne}$ and ${ }^{26} \mathrm{Mg}$ ). Right: same as the left panel but for three different densities in the H-box. The temperature is set to $50 \mathrm{MK}$.

the equilibrium value is quickly reached when the $\mathrm{CNO}$ cycle operates. After each injection of ${ }^{12} \mathrm{C}$, the equilibrium ratio is reached again almost instantaneously compared to the current timestep. In particular, $\left[{ }^{12} \mathrm{C} /{ }^{13} \mathrm{C}\right]$ reaches similar final values under all the explored temperatures and densities. When the $\mathrm{CNO}$ cycle operates, the $\left[{ }^{12} \mathrm{C} /{ }^{13} \mathrm{C}\right]$ equilibrium ratio is indeed almost temperature and density independent.

We see that the observed $[\mathrm{X} / \mathrm{Fe}]$ ratios are best covered when injecting ${ }^{12} \mathrm{C},{ }^{16} \mathrm{O}$, and ${ }^{22} \mathrm{Ne}$ (see the grey area in the third panel of Fig. 4). We note, however, that the models always give values that are too high for the $[\mathrm{Al} / \mathrm{Fe}]$ ratios (see Sect. 6). For all the other ratios, our very simple numerical experiment confirms the need for some mixing between the helium and hydrogen burning zones in the source star to explain the general pattern observed in CEMP-no stars in classes 2 and 4. The injection of ${ }^{22} \mathrm{Ne}$ seems to be needed, which supports the view that a strong mixing might be at work in the source star: ${ }^{22} \mathrm{Ne}$ can enter the hydrogen burning shell if ${ }^{12} \mathrm{C}$ and ${ }^{16} \mathrm{O}$ have first diffused in the hydrogen burning shell, but also if the created ${ }^{14} \mathrm{~N}$ have entered in turn in the helium burning core. For most of the ratios, however, we note that the grey region is wider than the ranges covered by the observations. We do not think this is a very serious problem when considering that CEMP-no stars are not made of pure hydrogen shell material. To be used to form new stars, this matter needs to be ejected either by winds or at the time of the supernova. In this process, the region where the CNO cycle is active (hydrogen shell) is mixed with other layers of the star and eventually with some interstellar medium. For instance, any mixing with the outer layers of the star where the iron has the same abundance as in the hydrogen shell - but where the CNO abundances are smaller and close to their initial values $\left(\sim 10^{-5}\right.$ for a model with $Z=10^{-5}$ ) - will shift the nitrogen abundance downward. Therefore, in order to obtain the observed nitrogen abundances in CEMP-no stars, it is likely needed that much higher abundances are reached in the hydrogen burning shell. While our box experiments provide some interesting constraints on the nuclear processes that might be needed to reproduce the peculiar abundance patterns of CEMP-no stars, only the computation of complete stellar models that take into account the ejection mechanism (both through winds and through the supernova explosion) and some possible mixing with the circumstellar material can provide abundances ratios that might be compared with the observed ratios in CEMP-no stars. This has to be kept in mind when interpreting the comparison shown in Fig. 4.

We note also that injecting some ${ }^{26} \mathrm{Mg}$ raises the $[\mathrm{Al} / \mathrm{Fe}]$ ratio far above the observed range. In stellar models, ${ }^{26} \mathrm{Mg}$ comes from the transformation of ${ }^{22} \mathrm{Ne}$ which occurs at the very end of the core helium burning phase. Thus, ${ }^{26} \mathrm{Mg}$ could be injected (if at all) only at the very end or after the core helium burning phase, leaving little time for nuclear burning to transform this ${ }^{26} \mathrm{Mg}$ into ${ }^{27} \mathrm{Al}$ in the hydrogen burning shell. In this respect, the present numerical experiments injecting ${ }^{26} \mathrm{Mg}$ regularly all along the burning of the hydrogen shell clearly overestimates what occurs in real stars. Thus, the hypothesis without ${ }^{26} \mathrm{Mg}$ injection is by far the most probable.

It is interesting to note that detailed stellar models are qualitatively well enough reproduced by this simple one-zone model. The two green patterns in Fig. 4 show the $[\mathrm{X} / \mathrm{Fe}]$ ratio in the hydrogen burning shell of a complete stellar model at the end of the core helium burning phase. The abundances are taken in the hydrogen shell where the energy released by hydrogen burning is the highest. The two stellar models are $20 M_{\odot}, Z=10^{-5}$ stars computed at $30 \%$ (second panel) and $70 \%$ (third panel) of the critical velocity on the ZAMS, which correspond to an initial equatorial velocity of 280 and $610 \mathrm{~km} \mathrm{~s}^{-1}$, respectively. Increasing the initial velocity can be modelled in the single-zone model by increasing the injection rate and injecting some ${ }^{22} \mathrm{Ne}$ in addition to the ${ }^{12} \mathrm{C}$ and ${ }^{16} \mathrm{O}$. By comparing the two green patterns, it is also interesting to see the strong impact of the initial rotation on the $[\mathrm{X} / \mathrm{Fe}]$ ratios in the hydrogen burning shell at core helium exhaustion. This shows that the stellar rotation at low metallicity is likely a non-negligible process.

One point that deserves more discussion is the constant value found for the ${ }^{12} \mathrm{C} /{ }^{13} \mathrm{C}$ ratio under various conditions. What can be learnt from this ratio? How can it be used in stellar evolution models? The lithium content at the surface of the CEMP-no stars can give interesting constraints as well. A deeper investigation into the $[\mathrm{Al} / \mathrm{Fe}]$ ratio also seems worthwhile because of the 
discrepancy we found between models and observations. Which conditions or assumptions will favour a lower $[\mathrm{Al} / \mathrm{Fe}]$ ratio, closer to the observed values?

\section{Dilution with material processed by helium burning and with initial ISM}

In the previous section we investigate the secular mixing between the hydrogen and helium burning regions during the nuclear life of the source star. We now discuss the mixing events that can happen outside the star when the nuclear reactions are no longer active. Different kinds of materials can be ejected by the source star into the ISM. We consider two of them here: (i) the material processed by hydrogen burning and (ii) the material processed by helium burning. Outside the star, these materials can be mixed together and/or with the ISM. We distinguish two kinds of dilutions:

1. the dilution between the material processed by hydrogen burning and the material processed by helium burning. This corresponds to a mixing between different parts of the star. By mixing we mean mixing of the ejecta (or stellar ejecta mixing, as defined in Sect. 2), hence outside of the star and without nuclear reactions.

2. the dilution between all the material ejected by the source star (processed by either hydrogen or helium burning) and the initial ISM, in which the source star formed. This is a mixing of the stellar ejecta with the initial ISM.

Based on considerations of the ${ }^{12} \mathrm{C} /{ }^{13} \mathrm{C}$ ratio and the lithium abundance, we investigate the possibility of constraining these two kinds of mixing events.

\subsection{Using the ${ }^{12} \mathrm{C} /{ }^{13} \mathrm{C}$ ratio to constrain the amount of ejecta processed by helium burning}

The ${ }^{12} \mathrm{C} /{ }^{13} \mathrm{C}$ ratio of the selected CEMP-no stars gives a strong constraint on the kind of material needed to form these stars. As we see in Fig. 4, this ratio is very close to the value found in a CNO processed material. We see from Figs. 4 and 5 that injecting some ${ }^{12} \mathrm{C}$ into a hydrogen burning region does not change the ${ }^{12} \mathrm{C} /{ }^{13} \mathrm{C}$ ratio under various densities, temperatures, and rates of injection. However, when the nuclear reactions are switched off (which is what happens when the material is ejected from the source star) and the CNO processed material is mixed with part of the region processed by helium burning, this ratio will no longer stay around the observed values.

This point can be illustrated with a simple experiment. It is possible to mix the two kinds of material ejected by the source star, the ejecta processed by hydrogen burning and by helium burning (hereafter $\mathrm{H}$-ejecta and He-ejecta). It is interesting to follow the evolution of the $\left[{ }^{12} \mathrm{C} /{ }^{13} \mathrm{C}\right]$ ratio as adding more and more He-ejecta to the H-ejecta. The left panel of Fig. 6 shows $\left[{ }^{12} \mathrm{C} /{ }^{13} \mathrm{C}\right]$ as a function of $f_{\text {mix }}$ defined as the fraction of Heejecta added to the $\mathrm{H}$-ejecta. For instance, $f_{\text {mix }}=10^{-2}$ means $1 \%$ of He-ejecta with $99 \%$ of $\mathrm{H}$-ejecta. We tested two compositions for the $\mathrm{H}$-ejecta. The first has mass fraction of ${ }^{12} \mathrm{C}$ and ${ }^{13} \mathrm{C}$ equal to $1.11 \times 10^{-7}$ and $3.10 \times 10^{-8}$. It is called $C$-poor H-ejecta. These mass fractions are the ones in the H-box at $t=0$. The second mixture has a ${ }^{12} \mathrm{C}$ mass fraction of $2.01 \times 10^{-4}$ and a ${ }^{13} \mathrm{C}$ mass fraction of $5.45 \times 10^{-5}$. We call it $C$-rich $H$-ejecta. These values correspond to the mass fractions of ${ }^{12} \mathrm{C}$ and ${ }^{13} \mathrm{C}$ in the H-box, a short time before hydrogen exhaustion when ${ }^{12} \mathrm{C}$ is injected.
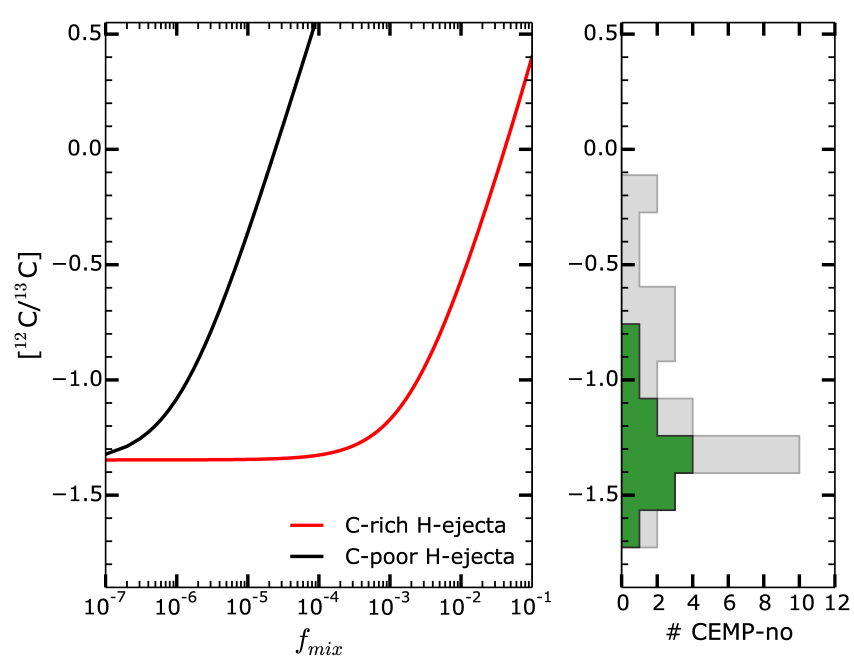

Fig. 6. Left: $\left[{ }^{12} \mathrm{C} /{ }^{13} \mathrm{C}\right]$ ratio as a function of the mixing factor $f_{\text {mix }}$, which represents the fraction of material processed by helium burning mixed with the material processed by hydrogen burning. Two compositions are tested for the material processed by hydrogen burning: a C-rich composition (red line) and a C-poor composition (black line). Right: green histogram showing the distribution of the CEMP-no stars used in this work. They belong to class 2 or 4 . The grey histogram shows all known CEMP-no stars with a measured ${ }^{12} \mathrm{C} /{ }^{13} \mathrm{C}$ ratio.

- The C-poor H-ejecta corresponds to the low rotational mixing case. A small amount of ${ }^{12} \mathrm{C}$ has diffused from the helium core to the hydrogen shell so that the mass fractions of ${ }^{12} \mathrm{C}$ and ${ }^{13} \mathrm{C}$ in the hydrogen burning shell stay around their initial value, i.e. around $10^{-7}$. When this part of the star is expelled, we get ejecta that is poor in carbon.

- The $C$-rich $H$-ejecta corresponds to the strong rotational mixing case. A lot of ${ }^{12} \mathrm{C}$ has diffused into the hydrogen burning shell, raising its abundance and that of ${ }^{13} \mathrm{C}$ far above their initial values. At the time of the ejection, this material is more enriched in carbon than in the previous case.

For the carbon abundances in the He-ejecta, we took the characteristic values in the helium burning core. We set $X_{\mathrm{Heb}}\left({ }^{12} \mathrm{C}\right)=$ 0.1 and $X_{\mathrm{Heb}}\left({ }^{13} \mathrm{C}\right)=0$ as a correct approximation.

We see in the left panel of Fig. 6 that mixing 1\% (i.e. $f_{\text {mix }}=10^{-2}$ ) of the He-ejecta with $99 \%$ of a C-rich H-ejecta leads to a $\left[{ }^{12} \mathrm{C} /{ }^{13} \mathrm{C}\right]$ of -0.6 , which lies above the range of observed values for the CEMP-no stars in classes 2 and 4 (see green histogram in left panel of Fig. 6 and Table 1). If no rotational mixing has occurred between the helium burning core and hydrogen burning shell of the source star, the H-ejecta is C-poor. In this case, mixing the same amount of He-ejecta as before with the $\mathrm{H}$-ejecta material leads to a $\left[{ }^{12} \mathrm{C} /{ }^{13} \mathrm{C}\right]$ ratio of 2.6 , far above the observed range. It seems that whatever the C-richness of the $\mathrm{H}$-ejecta, hence whatever the amount of helium products that enter the hydrogen burning shell during the life of the source star, the final contribution of the material processed by helium burning coming from the source star should be null to form the CEMP-no stars in classes 2 and 4. This strongly supports the idea that the CEMP-no stars in classes 2 and 4 are only made of the hydrogen envelope of the source star. More generally, since ${ }^{12} \mathrm{C} /{ }^{13} \mathrm{C}$ is highly sensitive to the burning region considered (it is low for a hydrogen burning region and high for helium burning), this ratio could be used to constrain the mass cut of spinstar models at the time of the supernova: the mass cut could be chosen in 
order to reproduce the observed ${ }^{12} \mathrm{C} /{ }^{13} \mathrm{C}$ ratio of the considered CEMP-no star.

The grey histogram in the right panel of Fig. 6 shows the distribution of all observed $\left[{ }^{12} \mathrm{C} /{ }^{13} \mathrm{C}\right]$ ratios at the surface of CEMP-no stars. It contains the 12 CEMP-no stars from Table 1 with a measured $\left[{ }^{12} \mathrm{C} /{ }^{13} \mathrm{C}\right]$ ratio plus 15 other CEMP-no stars. Some CEMP-no stars have higher $\left[{ }^{12} \mathrm{C} /{ }^{13} \mathrm{C}\right]$ ratios, suggesting the need for a small amount of material processed by helium burning to form them. We see, however, that the amount of material processed by helium burning needed should remain small in any case $\left(f_{\text {mix }} \lesssim 0.05\right)$ : mainly the hydrogen envelope of the progenitor should be used to form the CEMP-no stars. Nevertheless, we note that some CEMP-no stars only have a lower limit for the $\left[{ }^{12} \mathrm{C} /{ }^{13} \mathrm{C}\right]$ ratio (3 out of 12 in our subsample). An accurate determination of this ratio for those stars would be interesting in order to validate the previous statement regarding these stars.

\section{2. $A(L i)$ to constrain the dilution with the initial ISM}

In the previous section, we discussed the mixing of the stellar ejecta: the regions processed by hydrogen and helium burning ejected by the source star can be mixed together when the nuclear burning has stopped. The following discussion is related to the mixing (or the dilution) of the whole stellar ejecta with the initial ISM in which the source star formed.

Lithium is an interesting element that can be used to obtain information on the amount of ISM that should be mixed with the source star ejecta to form the CEMP-no stars. The abundance of lithium $A(\mathrm{Li})$ in the pristine ISM is equal to 2.72 according to Cyburt et al. (2008). It is totally destroyed in massive stars. As a consequence, as soon as the ejecta of the massive source star is mixed with the ISM, the abundance of lithium is raised (in the mixture made of initial ISM and source star ejecta). If we assume that the lithium content at the surface of the CEMP-no star reflects the lithium content in the cloud where it formed, then the higher the lithium content at the surface of the CEMP-no star, the more the source star ejecta was diluted with the ISM. The dilution factor for mixing the progenitor ejecta with the ISM can be chosen in order to obtain the observed $A(\mathrm{Li})$ value of the considered CEMP-no star.

One difficulty is that the lithium at the surface of the CEMP-no star can be depleted by internal mixing processes in the CEMP-no star itself. However, such processes might not be able to explain the low content of lithium observed at the surface of some CEMP-no stars. Meynet et al. (2010) pointed out that the maximal depletion predicted by the models of Korn et al. (2009) (1.2 dex) is unable to account for the $A(\mathrm{Li})$ value observed at the surface of HE 1327-2326 $(A(\mathrm{Li})<0.62$, see Table 1). According to the models of Korn et al. (2009), we would indeed expect a minimum observed value of $A(\mathrm{Li})=$ $2.72-1.2=1.52$ i.e. the WMAP content minus the maximum predicted depletion factor. We see that the depletion mechanism cannot easily account for the lowest observed $A(\mathrm{Li})$ values. The alternative for HE 1327-2326 is that it formed from Li-poor material.

The $A(\mathrm{Li})$ values (or upper limits) for nine of the considered CEMP-no stars are shown in Table 1. The lower panel of Fig. 3 in Meynet et al. (2010) shows the dilution factor $M_{\mathrm{ISM}} / M_{\text {eje }}$ vs. $A(\mathrm{Li})$. According to this figure and if we consider that the lithium was not depleted by the CEMP-no stars themselves, the dilution factor should be less than $\sim 0.1$ for the stars considered in this work. The highest dilution factor being for CS 22945-017, which has $A(\mathrm{Li})<1.51$. The final mass fraction of ${ }^{12} \mathrm{C},{ }^{14} \mathrm{~N}$, and ${ }^{16} \mathrm{O}$ in the H-box when injecting ${ }^{12} \mathrm{C}$ and ${ }^{16} \mathrm{O}$ is at least $10^{-3}$ (see Fig. 3). The mass fraction of the CNO elements in a $Z=10^{-5}$ ISM is about $10^{-6}$. Using $90 \%$ of $10^{-3}$ with $10 \%$ of $10^{-6}$ gives $\sim 10^{-3}$; the dilution does not play a significant role in this case.

We now suppose that the lithium was depleted by the CEMP-no stars. We take the maximum depletion factor (1.2 dex) from Korn et al. (2009) and we add it to the observed $A(\mathrm{Li})$ in order to get the initial $A(\mathrm{Li})$ value before the depletion process. The two highest $A(\mathrm{Li})$ belong to CS 22945-017 $(<2.71)$ and CS 22958-042 $(<2.53)$. Because $A(\mathrm{Li})$ is close to the WMAP value for CS 22945-017, it would imply a high dilution factor. However, for CS 22958-042 and all the other considered CEMP-no stars, the dilution factor should be less than $\sim 2$. There is still not enough ISM for the dilution to have a significant effect, except for CS 22945-017. Caution is needed when considering these simple statements about the dilution between ejecta and ISM, but in the framework of our simple model we see that the dilution with the initial ISM might only play a limited role because the metal abundances in the region processed by hydrogen burning are much higher than the values in the initial ISM and because the dilution factors derived from the lithium abundance are small in most of the cases.

\section{Al and Si abundances}

The experiments presented in Sect. 4 show that the $[\mathrm{Al} / \mathrm{Fe}]$ ratio in the H-box at hydrogen exhaustion always lies above the observed range of values. Even the initial $[\mathrm{Al} / \mathrm{Fe}]$ - taken from the hydrogen burning shell of a $60 M_{\odot}$ model at the beginning of the core helium burning phase - is just above the observed range (see lines with squares in Fig. 4). We investigate now three effects on the aluminium abundance: the injection (species and rate), the temperature, and the nuclear reaction rates.

\subsection{Impact of injection on Al: species and rate}

First of all, a lower final $[\mathrm{Al} / \mathrm{Fe}]$ ratio is expected if no ${ }^{26} \mathrm{Mg}$ is injected. If it is injected, some ${ }^{27} \mathrm{Al}$ is created through the reaction ${ }^{26} \operatorname{Mg}(p, \gamma)^{27} \mathrm{Al}$. This is illustrated in the third and fourth panels of Fig. 4. We see indeed that injecting ${ }^{26} \mathrm{Mg}$ leads to a higher final $[\mathrm{Al} / \mathrm{Fe}]$ than if no ${ }^{26} \mathrm{Mg}$ is injected.

Figure 7 shows $[\mathrm{Al} / \mathrm{Fe}]$ as a function of $R_{22 \mathrm{Ne}}$ : the rate of injection of ${ }^{22} \mathrm{Ne}$ at $T_{6}=50 \mathrm{MK}$ (left panel) and $T_{6}=80 \mathrm{MK}$ (right panel). The injected species are ${ }^{12} \mathrm{C},{ }^{16} \mathrm{O}$, and ${ }^{22} \mathrm{Ne}$. The blue, black, and green lines are associated with three sets of nuclear reactions rates that we tested and that will be discussed in Sect. 6.3. We focus on case 2 (black lines), which corresponds to the nuclear rates used here so far. The horizontal black line shows the initial $[\mathrm{Al} / \mathrm{Fe}]$ ratio in the $\mathrm{H}$-box and the black line with triangles shows the final values. We see that the final [Al/Fe] ratio at $T_{6}=80 \mathrm{MK}$ (right panel of Fig. 7) is lower when less ${ }^{22} \mathrm{Ne}$ is injected. When the injection rate is low enough, overall there is more ${ }^{27} \mathrm{Al}$ destroyed than created. We see that some aluminium is created at the end if $R_{22} \mathrm{Ne}>3 \times 10^{-11} M_{\odot} \mathrm{yr}^{-1}$ for the considered case. This aluminium comes mainly from the injected ${ }^{22} \mathrm{Ne}$ thanks to successive proton captures.

These two arguments suggest that a moderate amount of ${ }^{22} \mathrm{Ne}$ coming from the helium burning core to the hydrogen burning shell together with no ${ }^{26} \mathrm{Mg}$ would probably play in favour of a lower final $[\mathrm{Al} / \mathrm{Fe}]$ ratio.

\subsection{Impact of temperature on $A l$}

The final abundance of aluminium depends on the strength of the nuclear reactions rates that create and destroy it. The nuclear 
$\mathrm{T}_{6}=50 \mathrm{MK}$

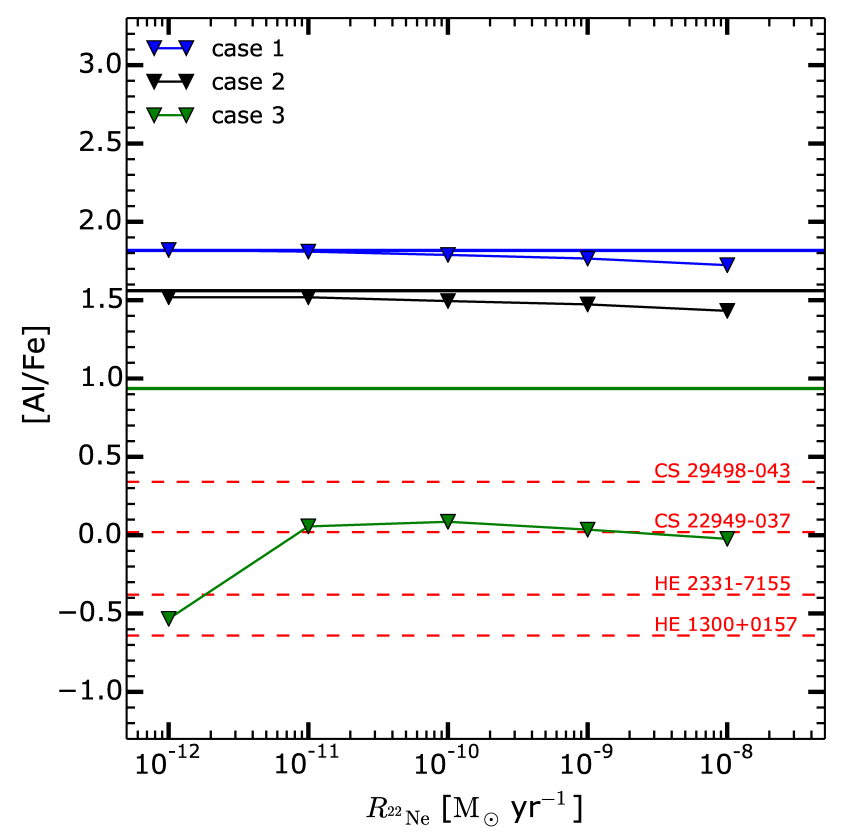

$\mathrm{T}_{6}=80 \mathrm{MK}$

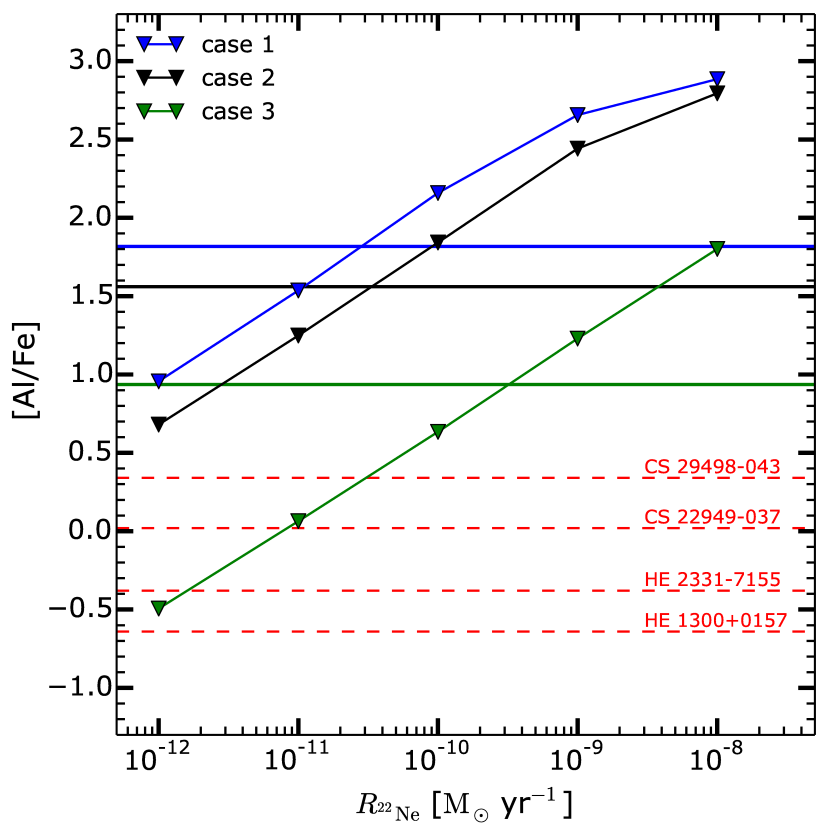

Fig. 7. $[\mathrm{Al} / \mathrm{Fe}]$ ratios in the $\mathrm{H}$-box as a function of $R^{22 \mathrm{Ne}}$, the rate of injection of ${ }^{22} \mathrm{Ne}\left({ }^{12} \mathrm{C}\right.$ and ${ }^{16} \mathrm{O}$ are also injected). The temperature is $T_{6}=50 \mathrm{MK}$ (left) and $T_{6}=80 \mathrm{MK}\left(\right.$ right); the density $\rho=1 \mathrm{~g} \mathrm{~cm}^{-3}$; and ${ }^{12} \mathrm{C},{ }^{16} \mathrm{O}$, and ${ }^{22} \mathrm{Ne}$ are injected. The three lines with triangles show the final $[\mathrm{Al} / \mathrm{Fe}]$ ratios in the $\mathrm{H}$-box when considering three different sets of nuclear rates for the three principal reactions implying ${ }^{27} \mathrm{Al}$ (see text for explanations). The horizontal blue, black, and green lines correspond to the initial $[\mathrm{Al} / \mathrm{Fe}]$ in the $\mathrm{H}$-box for the three considered cases. Observed $[\mathrm{Al} / \mathrm{Fe}]$ are shown by the red dashed lines for the CEMP-no stars that have $[\mathrm{Fe} / \mathrm{H}]=-3.8 \pm 0.3$.

rates for the $\mathrm{Ne}-\mathrm{Na}$ and $\mathrm{Mg}-\mathrm{Al}$ cycles are generally 3-4 dex higher at $T_{6}=80 \mathrm{MK}$ than at $T_{6}=50 \mathrm{MK}$ so that the synthesis of aluminium is slower at $T_{6}=50 \mathrm{MK}$. Figure 7 shows that indeed the final $[\mathrm{Al} / \mathrm{Fe}]$ ratios deviate very little from their initial values at $T_{6}=50 \mathrm{MK}$ (left panel), while the difference is much more significant at $T_{6}=80 \mathrm{MK}$ (right panel). We note however that the case 3 pattern at $T_{6}=50 \mathrm{MK}$ lies largely below its initial value (horizontal green line), but this is due to the ${ }^{26} \mathrm{Al}$ that has decayed into ${ }^{26} \mathrm{Mg}$ at the end of the simulation (this reduces $[\mathrm{Al} / \mathrm{Fe}]$ by about $1 \mathrm{dex}$ ). The green triangle at the abscissa $10^{-12} M_{\odot} \mathrm{yr}^{-1}$ also deviates from the others. The considered nuclear rates in this case disfavour the synthesis of ${ }^{27} \mathrm{Al}$ (see discussion in Sect. 6.3). This, together with the low injection rate that implies a longer time before hydrogen exhaustion, allows a larger depletion of ${ }^{27} \mathrm{Al}$ than at higher injection rates.

In addition, we saw in Sect. 4 (see also Fig. 5, left panel) that increasing the temperature leads to a lower final $[\mathrm{Al} / \mathrm{Fe}] \mathrm{ra}-$ tio (and a higher $[\mathrm{Si} / \mathrm{Fe}]$ ). This remains true as long as some ${ }^{26} \mathrm{Mg}$ is injected: if ${ }^{22} \mathrm{Ne}$ is injected but not ${ }^{26} \mathrm{Mg}$ (as in Fig. 7), $[\mathrm{Al} / \mathrm{Fe}]$ can be higher when increasing the temperature. This can be seen by comparing the left and right panels of Fig. 7 at the abscissa $10^{-9} M_{\odot} \mathrm{yr}^{-1}$ for instance. In this case, no ${ }^{26} \mathrm{Mg}$ is injected so that ${ }^{27} \mathrm{Al}$ comes mainly from the successive protons captured on the injected ${ }^{22} \mathrm{Ne}$. The chain leading to ${ }^{27} \mathrm{Al}$ is longer when starting from ${ }^{22} \mathrm{Ne}$ than from ${ }^{26} \mathrm{Mg}$. In that chain, the rate of ${ }^{24} \mathrm{Mg}(p, \gamma){ }^{25} \mathrm{Al}$ at $T_{6}=50 \mathrm{MK}$ is very low compared to the other reaction rates. This reaction tends to stop the chain at $T_{6}=50 \mathrm{MK}$ so that the final content in ${ }^{27} \mathrm{Al}$ is generally close to the initial one, even with high injection rates of ${ }^{22} \mathrm{Ne}$. Injecting some ${ }^{26} \mathrm{Mg}$ is a way to avoid this bottleneck reaction and synthesize some aluminium, even at $T_{6}=50 \mathrm{MK}$ (see the fourth panel of Fig. 4). The rate of ${ }^{24} \mathrm{Mg}(p, \gamma)^{25} \mathrm{Al}$ strongly increases from $T_{6}=50$ to $80 \mathrm{MK}$ (by about $7 \mathrm{dex}$ ) so that the chain leading to ${ }^{27} \mathrm{Al}$ (and ${ }^{28} \mathrm{Si}$ ) is no longer blocked at $T_{6}=80 \mathrm{MK}$.
A moderate temperature in the hydrogen shell ( 50 MK) is likely more compatible with a lower $[\mathrm{Al} / \mathrm{Fe}]$ under various amounts of ${ }^{22} \mathrm{Ne}$ coming from the helium core to the hydrogen shell. In the strong mixing case (high $R_{22} \mathrm{Ne}$ ) and at high temperatures, this could lead to a very high $[\mathrm{Al} / \mathrm{Fe}]$ ratio (Fig. 7 , right panel).

\subsection{Impact of changing the nuclear rates on Al}

A point that deserves to be investigated is the uncertainties of the nuclear rates. In a hydrogen burning region, the two reactions destroying ${ }^{27} \mathrm{Al}$ are ${ }^{27} \mathrm{Al}(p, \gamma)^{28} \mathrm{Si}$ and ${ }^{27} \mathrm{Al}(p, \alpha){ }^{24} \mathrm{Mg}$. The reaction which creates ${ }^{27} \mathrm{Al}$ is ${ }^{26} \mathrm{Mg}(p, \gamma){ }^{27} \mathrm{Al}$. The three nuclear rates associated with these reactions are very uncertain at the considered temperatures. To illustrate this point, we compared three sets of nuclear rates for the three reactions involving ${ }^{27} \mathrm{Al}$. We used rates provided by the JINA REACLIB database (Cyburt et al. 2010).

- Case 1: the best scenario for the ${ }^{27} \mathrm{Al}$ synthesis. We took the maximum rate (at $T_{6}=80$ ) for ${ }^{26} \mathrm{Mg}(p, \gamma){ }^{27} \mathrm{Al}$ (Cyburt et al. 2010). The minimum rate was taken for both ${ }^{27} \mathrm{Al}(p, \gamma)^{28} \mathrm{Si}$ and ${ }^{27} \mathrm{Al}(p, \alpha){ }^{24} \mathrm{Mg}$ (van Wormer et al. 1994).

- Case 2: we used the rates taken from this work (see Sect. 3).

- Case 3: the best scenario for ${ }^{27} \mathrm{Al}$ destruction. The minimum rate for ${ }^{26} \mathrm{Mg}(p, \gamma)^{27} \mathrm{Al}$ is from Angulo et al. (1999) and the maximum rates for ${ }^{27} \mathrm{Al}(p, \gamma){ }^{28} \mathrm{Si}$ and ${ }^{27} \mathrm{Al}(p, \alpha){ }^{24} \mathrm{Mg}$ are from Cyburt et al. (2010).

To be consistent, we computed two other complete stellar models from the ZAMS to core helium burning ignition with the two new sets of nuclear rates (cases 1 and 3). Depending on the set of nuclear rates chosen, the initial abundances in the H-box are taken from the hydrogen burning shell of the corresponding stellar model. The three initial $[\mathrm{Al} / \mathrm{Fe}]$ ratios taken in the $\mathrm{H}$-box are 
shown by the blue (case 1), black (case 2), and green (case 3) horizontal lines. We see that the scatter is significant. This occurs because the $\mathrm{Mg}-\mathrm{Al}$ cycle is already operating in the core of the complete stellar model during the main sequence so that the aluminium abundance is affected if the nuclear rates are changed. Depending on the set of nuclear rates, it finally leads to a different aluminium content in the hydrogen shell at core helium burning ignition, hence in the H-box.

The lines with triangles correspond to the final $[\mathrm{Al} / \mathrm{Fe}]$ ratios in the H-box for the three cases. We verify that the rates considered in this work (case 2) lead to a final $[\mathrm{Al} / \mathrm{Fe}]$ ratios that is between the two extreme cases. Whatever the injection rate, at least 1.5 dex separates the blue pattern from the green; the green pattern gives lower $[\mathrm{Al} / \mathrm{Fe}]$ since this is the case where ${ }^{27} \mathrm{Al}$ is the most destroyed and the least synthesized. A word of caution: for case 3 , the abundance of ${ }^{26} \mathrm{Al}$ is higher than that of ${ }^{27} \mathrm{Al}$ during the burning, so that $[\mathrm{Al} / \mathrm{Fe}]$ is significantly affected when ${ }^{26} \mathrm{Al}$ decays into ${ }^{26} \mathrm{Mg}$. For cases 1 and $2,{ }^{27} \mathrm{Al}$ is more abundant so that decaying ${ }^{26} \mathrm{Al}$ at the end reduces $[\mathrm{Al} / \mathrm{Fe}]$ only a little.

Four out of five CEMP-no stars with $[\mathrm{Fe} / \mathrm{H}]=-3.8 \pm 0.3$ have an observed $[\mathrm{Al} / \mathrm{Fe}]$. These ratios are represented by the red dashed lines in Fig. 7. The scatter of the observed [Al/Fe] ratios is covered well enough by case 3 (green pattern) when relying on different values of $R_{22} \mathrm{Ne}$, the injection rate of ${ }^{22} \mathrm{Ne}$. If we select that set of rates, our model indicates that (i) the CEMP-no stars with $[\mathrm{Al} / \mathrm{Fe}] \gtrsim 0$ could be made of a material processed by hydrogen burning at relatively high temperatures (about $80 \mathrm{MK}$ ) because $T \sim 50 \mathrm{MK}$ would not lead to a high enough aluminium content (see left panel of Fig. 7) and (ii) only a high enough injection rate of ${ }^{22} \mathrm{Ne}$ can account for Al-enhanced CEMP-no stars. The four stars considered here do not show such a high [Al/Fe] ratio (except a modest enhancement in CS 29498-043). This might tend to disfavour the progenitors where the mixing is very strong (very high initial velocity) and with a high temperature in the hydrogen shell ( $\gtrsim 60 M_{\odot}$ stars). The two remaining possibilities are either a high temperature $(\sim 80 \mathrm{MK})$ in the hydrogen shell but a weak mixing (a low $R_{22 \mathrm{Ne}}$ ), or a moderate temperature in the hydrogen shell $(\sim 50 \mathrm{MK})$ with a weak to strong mixing. A moderate temperature in the hydrogen shell is more likely achieved in $\sim 20 M_{\odot}$ progenitors $(30-60 \mathrm{MK}$ ) rather than in $\sim 60 M_{\odot}$ ones (30-80 MK, see Fig. 2).

\subsection{Sensitivity of the Si abundance}

The silicon abundance is also affected when changing the injection, temperature, or nuclear reaction rates. In the explored range of parameters (temperature, injection rate, and nuclear reaction rates; see Fig. 7) and for the injected species considered $\left({ }^{12} \mathrm{C}\right.$, ${ }^{16} \mathrm{O}$, and $\left.{ }^{22} \mathrm{Ne}\right)$, the final $[\mathrm{Si} / \mathrm{Fe}]$ ranges from 1 to 1.8 .

At $T_{6}=50 \mathrm{MK}$, the final $[\mathrm{Si} / \mathrm{Fe}]$ ratio depends very weakly on the injection rate because the $\mathrm{Ne}-\mathrm{Si}$ chain is stopped by the ${ }^{24} \mathrm{Mg}(p, \gamma)^{25} \mathrm{Al}$ reaction (see Sect. 6.2). In this case, the final [Si/Fe] ratio is almost equal to the initial value, which ranges from 1 (case 1 , lowest rate for ${ }^{27} \mathrm{Al}(p, \gamma){ }^{28} \mathrm{Si}$ ) to 1.2 (case 3, highest rate for $\left.{ }^{27} \mathrm{Al}(p, \gamma){ }^{28} \mathrm{Si}\right)$.

At $T_{6}=80 \mathrm{MK}$, the final abundance of silicon is always enhanced compared to the initial level. The ${ }^{24} \mathrm{Mg}(p, \gamma)^{25} \mathrm{Al}$ reaction no longer blocks the $\mathrm{Ne}-\mathrm{Si}$ chain so that the injected ${ }^{22} \mathrm{Ne}$ synthesizes some ${ }^{28} \mathrm{Si}$. The final [Si/Fe] ratios range between 1.2 and 1.8 .

Three CEMP-no stars with $[\mathrm{Fe} / \mathrm{H}]=-3.8 \pm 0.3$ have a measured $[\mathrm{Si} / \mathrm{Fe}]$. The values are $0.77,0.82$, and 0.87 . These values are closer to the results given by the model at $T_{6}=50 \mathrm{MK}$ $(1<[\mathrm{Si} / \mathrm{Fe}]<1.2)$, which might indicate a moderate temperature in hydrogen shell of the progenitor $\left(T_{6} \simeq 50 \mathrm{MK}\right)$, consistent with the discussion in Sect. 6.3.

\section{Possible astronomical sources of classes 2 and 4 CEMP-no stars}

Through the present work, we suggest that the high observed abundances of $\mathrm{C}$ together with that of $\mathrm{N}, \mathrm{O}, \mathrm{Na}$, and $\mathrm{Mg}$ at the surface of the CEMP-no stars are the signatures of a mixing between the helium and hydrogen burning regions of the source star during its nuclear life. What objects are able to experience such a mixing process? In the framework of our results, but also in a more global context, we speculate on the possible progenitors of classes 2 and 4 CEMP-no stars.

\section{1. $A G B$ stars}

AGB stars are known contributors to s-process elements. They are generally believed to be responsible for the enrichment, by mass transfer, in s-elements observed at the surface of the CEMP-s stars. In addition to the abundances, the models have to reproduce the period of the binary system for instance, which can give tight contraints but also increase the difficulty of finding models matching the observations (see e.g. Abate et al. 2015).

In AGB stars, there is a mixing between the two shells. It could also, in principle, be enhanced by rotation or, at the very least, rotation may change the chemical structure of the star at the beginning of the AGB phase (see e.g. the $7 M_{\odot}$ model in Meynet et al. 2010).

It seems however that there are at least two difficulties in this scenario to explain the CEMP-no stars. First, the AGB stars are experiencing the s-process and by definition, the CEMP-no class is not enriched in s-elements (or is weakly enriched). This feature would be difficult to explain relying on AGB stars. Second, it seems difficult to account for the CEMP-no stars having $[\mathrm{Fe} / \mathrm{H}] \lesssim-4$ with the AGB stars: such a low iron content likely indicates that only the most massive objects, more massive than the AGB stars, have contributed.

\subsection{Faint supernovae, mixing and fallback}

Tominaga et al. (2014) discussed the scenario of faint supernovae from Pop III stars with mixing and fallback. In these models, only the outer layers are ejected from the progenitor. It is indeed needed to explain the observed $\mathrm{CNO}$ abundance patterns, as well as the low ${ }^{12} \mathrm{C} /{ }^{13} \mathrm{C}$ ratios. This is in line with the discussion about the mixing of the ejecta; we see in Sect. 5 that little or no material processed by helium burning coming from the source star should be mixed into the hydrogen rich envelope when the nuclear life of the star is finished. In other words, mainly the hydrogen rich envelope of the progenitor would be needed to form the future CEMP-no star.

On the other hand, these models are generally non-rotating, leading to some difficulties in explaining the high nitrogen abundance observed in some CEMP-no stars without invoking an extra mixing process in the progenitor. Rotating models were however considered in Takahashi et al. (2014), predicting higher $\mathrm{N} / \mathrm{Fe}$ ratios in the ejecta, almost in line with the observed values at the surface of two out of the three CEMP-no stars they considered (HE 0107-5240 and HE 1327-2326).

\subsection{Contribution of more than one source}

Limongi et al. (2003) proposed a two-step scenario: a normal supernova from a $\sim 15 M_{\odot}$ progenitor responsible for the iron-peak 
elements, followed by a fainter one experiencing strong fallback coming from a $\sim 35 M_{\odot}$ progenitor. The second more massive progenitor enriches the ISM in light elements: $\mathrm{C}, \mathrm{N}, \mathrm{Na}$, and $\mathrm{Mg}$. These elements are produced thanks to a partial mixing between hydrogen and helium burning shells that can occur in $Z=0$ models, even in non-rotating models.

What seems to not match with the CEMP-no stars in classes 2 and 4 is the predicted ${ }^{12} \mathrm{C} /{ }^{13} \mathrm{C}$ ratio of 240 for this twostep model. The corresponding $\left[{ }^{12} \mathrm{C} /{ }^{13} \mathrm{C}\right]$ ratio is 0.4 and this is not compatible with the values of the CEMP-no stars considered here (see Fig. 6, right panel). We note, however, that 3 out of 12 have only a lower limit for the ${ }^{12} \mathrm{C} /{ }^{13} \mathrm{C}$ ratio (see Table 1 ) so that such a high predicted ${ }^{12} \mathrm{C} /{ }^{13} \mathrm{C}$ could be consistent with these CEMP-no stars.

\subsection{Spinstars}

Several signatures of fast rotation at low metallicity have been found over the past years. One strong signature is that the high nitrogen abundances and the low ${ }^{12} \mathrm{C} /{ }^{13} \mathrm{C}$ ratios observed in normal very metal-poor (VMP) stars are much better reproduced by low metallicity chemical evolution models when including fast rotators, also called spinstars (Chiappini et al. 2006, 2008). Because of the high rotation, the injection process we investigate here operates in the spinstar and it could be a way to obtain material enriched in $\mathrm{C}, \mathrm{N}, \mathrm{O}, \mathrm{Na}$, and $\mathrm{Mg}$, together with a low ${ }^{12} \mathrm{C} /{ }^{13} \mathrm{C}$, that will ultimately form a CEMP-no star in class 2 or 4.

The spinstars and more generally the objects experiencing mixing between hydrogen and helium burning regions appear to be interesting progenitor candidates for class 2 and 4 CEMP-no stars.

\section{Conclusions}

We studied the possibility of forming CEMP-no stars with a material processed by hydrogen burning coming from the source star. We carried out nuclear experiments where the convective hydrogen burning shell of the source star was modelled by a hydrogen burning single zone (H-box). The mixing between the helium burning core and the hydrogen burning shell was mimicked by injecting the products of helium burning ${ }^{12} \mathrm{C},{ }^{16} \mathrm{O},{ }^{22} \mathrm{Ne}$, and ${ }^{26} \mathrm{Mg}$ in the H-box. When injecting these species in the hydrogen burning zone ${ }^{14} \mathrm{~N},{ }^{23} \mathrm{Na},{ }^{24} \mathrm{Mg}$, and ${ }^{27} \mathrm{Al}$ are synthesized. The ${ }^{12} \mathrm{C} /{ }^{13} \mathrm{C}$ ratio is constant under various densities, temperatures in the H-box, and also under various injection rates. The $[\mathrm{Al} / \mathrm{Fe}]$ ratio in the hydrogen burning zone lies generally above the observations. Using different nuclear reaction rates found in the literature for the reactions involving ${ }^{27} \mathrm{Al}$ leads to a better coverage of the observed $[\mathrm{Al} / \mathrm{Fe}]$ scatter. The high observed $[\mathrm{Al} / \mathrm{Fe}]$ ratios are reproduced at sufficiently high hydrogen burning temperature $(80 \mathrm{MK})$ and if the injection rate of ${ }^{22} \mathrm{Ne}$ is high enough. This might point toward a massive (high temperature) and fast rotating (high injection rate) progenitor.

Throughout this work, we suggest that the high observed abundances of light elements at the surface of the CEMP-no stars are the signatures of a mixing between the helium and hydrogen burning regions of the progenitor during its nuclear life. It supports the CEMP-no star formation scenario of Maeder \& Meynet (2015) for classes 2 and 4. This scenario states that these stars are made of a material processed by hydrogen burning only, but were products of helium burning coming from the helium core of the source star diffused into the hydrogen burning shell thanks to the rotational mixing. This arrival of new elements boosts the nucleosynthesis in the hydrogen burning shell. Considerations on the ${ }^{12} \mathrm{C} /{ }^{13} \mathrm{C}$ ratio confirmed that the CEMP-no stars in classes 2 and 4 are made of a material that was only processed by hydrogen burning in the source star. This corroborates the assumption that the CEMP-no stars formed mainly with the hydrogen-rich envelope of the source star. The ${ }^{12} \mathrm{C} /{ }^{13} \mathrm{C}$ ratio is highly sensitive to the burning region considered in the source star (hydrogen or helium burning region). It could be used to constrain the part expelled from the source star at the time of the supernova in order to reproduce the observed ${ }^{12} \mathrm{C} /{ }^{13} \mathrm{C}$ ratio at the surface of the CEMP-no stars.

The spinstars are interesting candidates for being the class 2 and 4 CEMP-no progenitors because of their rotation which induces exchanges of material between the hydrogen and helium burning regions. This gives some support to the idea that the rotation played an important role in the early chemical evolution of galaxies.

Acknowledgements. The authors thank the anonymous referee who helped to improve this paper through very constructive remarks. This work was supported by the Swiss National Science Foundation (project number 200020-160119).

\section{References}

Abate, C., Pols, O. R., Karakas, A. I., \& Izzard, R. G. 2015, A\&A, 576, A118

Allen, D. M., Ryan, S. G., Rossi, S., Beers, T. C., \& Tsangarides, S. A. 2012, A\&A, 548, A34

Angulo, C., Arnould, M., Rayet, M., et al. 1999, Nucl. Phys. A, 656, 3

Aoki, W. 2010, in IAU Symp. 265, eds. K. Cunha, M. Spite, \& B. Barbuy, 111

Aoki, W., Beers, T. C., Christlieb, N., et al. 2007, ApJ, 655, 492

Beers, T. C., \& Christlieb, N. 2005, ARA\&A, 43, 531

Carollo, D., Beers, T. C., Bovy, J., et al. 2012, ApJ, 744, 195

Chiappini, C. 2013, Astron. Nachr., 334, 595

Chiappini, C., Hirschi, R., Meynet, G., et al. 2006, A\&A, 449, L27

Chiappini, C., Ekström, S., Meynet, G., et al. 2008, A\&A, 479, L9

Choplin, A., Meynet, G., Maeder, A., Hirschi, R., \& Chiappini, C. 2016, ArXiv e-prints [arXiv: 1602.04122]

Cyburt, R. H., Fields, B. D., \& Olive, K. A. 2008, J. Cosmol. Astropart. Phys., 11,012

Cyburt, R. H., Amthor, A. M., Ferguson, R., et al. 2010, ApJS, 189, 240

Ekström, S., Georgy, C., Eggenberger, P., et al. 2012, A\&A, 537, A146

Frebel, A., Christlieb, N., Norris, J. E., et al. 2006, ApJ, 652, 1585

Frebel, A., Norris, J. E., Aoki, W., et al. 2007, ApJ, 658, 534

Frebel, A., Collet, R., Eriksson, K., Christlieb, N., \& Aoki, W. 2008, ApJ, 684 588

Hale, S. E., Champagne, A. E., Iliadis, C., et al. 2002, Phys. Rev. C, 65, 015801

Hansen, T., Hansen, C. J., Christlieb, N., et al. 2014, ApJ, 787, 162

Hansen, T., Hansen, C. J., Christlieb, N., et al. 2015, ApJ, 807, 173

Hirschi, R. 2007, A\&A, 461, 571

Iliadis, C., D’Auria, J. M., Starrfield, S., Thompson, W. J., \& Wiescher, M. 2001, ApJS, 134, 151

Keller, S. C., Bessell, M. S., Frebel, A., et al. 2014, Nature, 506, 463

Korn, A. J., Richard, O., Mashonkina, L., et al. 2009, ApJ, 698, 410

Lee, Y. S., Beers, T. C., Masseron, T., et al. 2013, AJ, 146, 132

Limongi, M., Chieffi, A., \& Bonifacio, P. 2003, ApJ, 594, L123

Lodders, K. 2003, ApJ, 591, 1220

Maeder, A., \& Meynet, G. 2001, A\&A, 373, 555

Maeder, A., \& Meynet, G. 2015, A\&A, 580, A32

Maeder, A., Meynet, G., \& Chiappini, C. 2015, A\&A, 576, A56

Masseron, T., van Eck, S., Famaey, B., et al. 2006, A\&A, 455, 1059

Masseron, T., Johnson, J. A., Plez, B., et al. 2010, A\&A, 509, A93

Masseron, T., Johnson, J. A., Lucatello, S., et al. 2012, ApJ, 751, 14

Meynet, G., Ekström, S., \& Maeder, A. 2006, A\&A, 447, 623

Meynet, G., Hirschi, R., Ekstrom, S., et al. 2010, A\&A, 521, A30

Norris, J. E., Yong, D., Bessell, M. S., et al. 2013, ApJ, 762, 28

Placco, V. M., Frebel, A., Beers, T. C., \& Stancliffe, R. J. 2014, ApJ, 797, 21

Roederer, I. U., Preston, G. W., Thompson, I. B., et al. 2014, AJ, 147, 136

Takahashi, K., Umeda, H., \& Yoshida, T. 2014, ApJ, 794, 40

Tominaga, N., Iwamoto, N., \& Nomoto, K. 2014, ApJ, 785, 98

Umeda, H., \& Nomoto, K. 2002, ApJ, 565, 385

Umeda, H., \& Nomoto, K. 2005, ApJ, 619, 427

van Wormer, L., Görres, J., Iliadis, C., Wiescher, M., \& Thielemann, F.-K. 1994, ApJ, 432, 326 


\section{Appendix A}

Let us consider a box of initial mass $m=1 M_{\odot}$, where $X_{i}$ denotes the mass fraction of the element $i$. We also consider a reservoir composed only of the element $e$, so that its mass fraction $X_{e}^{\prime}$ in the reservoir is 1 . During a time $\Delta t$, we inject a mass

$\Delta m=R_{e} \Delta t$

from the reservoir into the box. $R_{e}$ is the injection rate of the element $e$ expressed in $M_{\odot} \mathrm{yr}^{-1}$. After the injection, the new mass fraction $X_{e}^{\text {new }}$ of the injected element in the box is

$X_{e}^{\text {new }}=\frac{X_{e} m+X_{e}^{\prime} \Delta m}{m+\Delta m}=\frac{X_{e} m+R_{e} \Delta t}{m+R_{e} \Delta t}$.

The new mass fraction of the other elements in the box can be expressed as

$X_{i \neq e}^{\text {new }}=\frac{X_{i} m}{m+R_{e} \Delta t}$.

We note that the H-box that was initially $1 M_{\odot}$ grows in mass as a result of the injection. Its final mass is generally similar to the initial one and never exceeds $\sim 1.3 M_{\odot}$ for the presented results, which stays relatively close to $1 M_{\odot}$.

The point is now to estimate $R_{e}$ the injection rate. Let us consider the example of the carbon. In stellar models, the primary ${ }^{14} \mathrm{~N}$ is synthesized through the diffusion of ${ }^{12} \mathrm{C}$ and ${ }^{16} \mathrm{O}$ from the helium core to the hydrogen burning shell. The secondary ${ }^{14} \mathrm{~N}$ is formed with the initial CNO elements in the star. It is possible to roughly quantify $M_{14}^{\text {prim }}$ the mass fraction of primary ${ }^{14} \mathrm{~N}$ formed during the core helium burning phase as

$M_{{ }^{\mathrm{p}} \mathrm{N}}^{\mathrm{prim}}=\left(\int_{0}^{M} X_{{ }^{14} \mathrm{~N}}\left(M_{r}\right) \mathrm{d} M_{r}\right)_{Y_{\mathrm{c}}=0}-\left(X_{\mathrm{C}, \text { ini }}+X_{\mathrm{N}, \text { ini }}+X_{\mathrm{O}, \text { ini }}\right) M$, where $Y_{\mathrm{c}}$ is the central ${ }^{4} \mathrm{He}$ mass fraction; $X_{{ }^{14} \mathrm{~N}}\left(M_{r}\right)$ the mass fraction of ${ }^{14} \mathrm{~N}$ at coordinate $M_{r} ; X_{\mathrm{C} \text {,ini }}, X_{\mathrm{N}, \text { ini }}, X_{\mathrm{O} \text {,ini }}$ the mass fractions of the CNO elements at the ZAMS; and $M$ the total mass of the star at the end of the core helium burning phase. $M_{{ }_{14} \mathrm{~N}}^{\text {prim }}$ is defined as the total amount of ${ }^{14} \mathrm{~N}$ in the star at core helium exhaustion minus the amount of ${ }^{14} \mathrm{~N}$ that can be formed with the initial CNO content (secondary ${ }^{14} \mathrm{~N}$ ). We suppose that all the ${ }^{12} \mathrm{C}$ and ${ }^{16} \mathrm{O}$ diffusing from the helium core to the hydrogen shell are transformed into ${ }^{14} \mathrm{~N}$. In this case, to get a mass $M_{{ }_{14} \mathrm{~N}}^{\text {prim }}$ of primary nitrogen in the star at the end of core helium burning, an average injection rate of $\left({ }^{12} \mathrm{C}+{ }^{16} \mathrm{O}\right)$ is needed in the hydrogen shell of

$R^{12} \mathrm{C}+{ }^{16} \mathrm{O}=\frac{M_{14}^{\mathrm{N}}}{\tau_{\mathrm{HeB}}}$,

where $\tau_{\mathrm{HeB}}$ is the duration of the core helium burning phase. For a $60 M_{\odot}$ model at $Z=10^{-5}$ and at $70 \%$ of the critical velocity at the ZAMS, we find $R_{{ }^{12} \mathrm{C}+{ }^{16} \mathrm{O}}=5 \times 10^{-8} M_{\odot} \mathrm{yr}^{-1}$.

The amount of primary nitrogen synthesized (hence the value of $R^{12} \mathrm{C}+{ }^{16} \mathrm{O}$ ) can change significantly depending on the rotation, the mass of the model, or the prescription for the rotational mixing for instance. In the present work we consider $10^{-10}<R_{12} \mathrm{C}<10^{-6} M_{\odot} \mathrm{yr}^{-1}$ (the chosen values are $10^{-10}$, $10^{-8}$, and $\left.10^{-6} M_{\odot} \mathrm{yr}^{-1}\right)$. In addition, we set $R_{{ }^{12} \mathrm{C}}=R_{{ }_{16} \mathrm{O}}$ and $R_{22 \mathrm{Ne}}=R_{26 \mathrm{Mg}}=R_{{ }^{12} \mathrm{C}} / 100$. The factor 100 between the two rates accounts at first order for the fact that ${ }^{22} \mathrm{Ne}$ and ${ }^{26} \mathrm{Mg}$ are $\sim 100$ times less abundant than ${ }^{12} \mathrm{C}$ and ${ }^{16} \mathrm{O}$ in the helium burning core of a low metallicity massive stellar model, so that $\sim 100$ times less ${ }^{22} \mathrm{Ne}$ and ${ }^{26} \mathrm{Mg}$ will enter by rotational mixing into the hydrogen burning shell. 